\begin{tabular}{|c|l|}
\hline Title & Stokes and Navier-Stokes equations with Robin boundary conditions in a half-space \\
\hline Author(s) & Saal, Juergen \\
\hline Citation & Hokkaido University Preprint Series in Mathematics, 633, 1-29 \\
\hline Issue Date & 2004 \\
\hline DOI & 10.14943/83786 \\
\hline Doc URL & http://hdl.handle.net/2115/69440 \\
\hline Type & bulletin (article) \\
\hline File Information & pre633.pdf \\
\hline
\end{tabular}

Instructions for use 


\title{
Stokes and Navier-Stokes equations with Robin boundary conditions in a half-space
}

\author{
Jürgen Saal*
}

\begin{abstract}
We study the initial-boundary value problem for the Stokes equations with Robin boundary conditions in the half-space $\mathbb{R}_{+}^{n}$. It is proved that the associated Stokes operator is sectorial and admits a bounded $H^{\infty}$-calculus on $L_{\sigma}^{q}\left(\mathbb{R}_{+}^{n}\right)$. As an application we prove also a local existence result for the nonlinear initial value problem of the Navier-Stokes equations with Robin boundary conditions.
\end{abstract}

\section{Introduction}

In this article we consider the Stokes equations of the type

$$
\left\{\begin{aligned}
\partial_{t} u-\Delta u+\nabla p & =f \quad \text { in } \mathbb{R}_{+}^{n} \times(0, \infty) \\
\operatorname{div} u & =0 \quad \text { in } \mathbb{R}_{+}^{n} \times(0, \infty), \\
u(0) & =u_{0} \text { in } \mathbb{R}_{+}^{n} \\
T_{\alpha} u & =0 \quad \text { in } \partial \mathbb{R}_{+}^{n} \times(0, \infty)
\end{aligned}\right.
$$

with velocity field $u$ and pressure $p$ in the Lebesgue space $L^{q}\left(\mathbb{R}_{+}^{n}\right)$ for $1<q<\infty$. The trace operator $T_{\alpha}$ here is given by Robin boundary conditions, i.e.

$$
T_{\alpha} u:=\left.\left(\begin{array}{c}
\alpha u^{\prime}-\partial_{n} u^{\prime} \\
u^{n}
\end{array}\right)\right|_{\partial \mathbb{R}_{+}^{n}},
$$

where $u^{\prime}$ denotes the tangential part of $u$ and $\alpha \in[0, \infty]^{1}$. Observe, that the case $\alpha=0$ or $\alpha=\infty$ corresponds to the classical Neumann or Dirichlet boundary conditions respectively.

We will give a comprehensive discussion of the properties of $A_{\alpha}$, the associated Stokes operator with Robin boundary conditions. As an application of the results for the Stokes operator we further will prove a local existence result for the Navier-Stokes equations with Robin boundary conditions, i.e. for the nonlinear system

$$
\left\{\begin{aligned}
\partial_{t} u-\Delta u+(u \cdot \nabla) u+\nabla p & =0 \text { in } \mathbb{R}_{+}^{n} \times(0, T), \\
\operatorname{div} u & =0 \text { in } \mathbb{R}_{+}^{n} \times(0, T), \\
u(0) & =u_{0} \text { in } \mathbb{R}_{+}^{n}, \\
T_{\alpha} u & =0 \text { in } \partial \mathbb{R}_{+}^{n} \times(0, T) .
\end{aligned}\right.
$$

${ }^{*}$ COE Post Doctoral Fellow at Hokkaido University, Sapporo

${ }^{1}$ The case $\alpha=\infty$ is to understand in the following sense: divide the first line in (2) by $\alpha$ and let $\alpha \rightarrow \infty$. 
We start our discussion for the Stokes operator $A_{\alpha}$ by constructing an explicit solution formula for the Stokes resolvent problem with Robin boundary conditions in the half-space $\mathbb{R}_{+}^{n}$. Based on this formula we will prove resolvent estimates, which imply, that the Stokes operator is the generator of a bounded holomorphic strongly continuous semigroup in the solenoidal space $L_{\sigma}^{q}\left(\mathbb{R}_{+}^{n}\right)$. Moreover, this semigroup satisfies $L^{p}-L^{q}$ estimates which are stated for holomorphic semigroups in $L^{q}$-spaces in a more general framework in a previous section. Next we show that the Stokes operator with Robin boundary conditions even admits a bounded $H^{\infty}$-calculus on $L_{\sigma}^{q}\left(\mathbb{R}_{+}^{n}\right)$. Hence, according to the results in [13], $A_{\alpha}$ has maximal regularity, i.e. the solution of problem (1) satisfies the estimate

$$
\int_{0}^{\infty}\left(\left\|u_{t}(t)\right\|_{q}^{p}+\left\|\nabla^{2} u(t)\right\|_{q}^{p}+\|\nabla p(t)\|_{q}^{p}\right) \mathrm{d} t \leq C\left(\left\|u_{0}\right\|_{\left(L_{\sigma}^{q}\left(\mathbb{R}_{+}^{n}\right), D\left(A_{\alpha}\right)\right)_{1-\frac{1}{q}, q}}+\int_{0}^{\infty}\|f(t)\|_{q}^{p} \mathrm{~d} t\right)
$$

for all $f \in L^{p}\left((0, \infty), L_{\sigma}^{q}\left(\mathbb{R}_{+}^{n}\right)\right)$ and $u_{0} \in\left(L_{\sigma}^{q}\left(\mathbb{R}_{+}^{n}\right), D\left(A_{\alpha}\right)\right)_{1-\frac{1}{q}, q}$, where $1<p, q<\infty$.

It is known that the class of all operators admitting a bounded $H^{\infty}$-calculus coincides with the (a priori smaller) class of all operators admitting an R-bounded $H^{\infty}$-calculus if the underlying Banach space has property $(\alpha)$, see [16] and [4]. Since the space $L_{\sigma}^{q}(\Omega)$ is known to enjoy this property for any domain $\Omega$ and any $q \in[1, \infty]$, we can immediately conclude that the Stokes operator with Robin boundary conditions even admits an R-bounded $H^{\infty}$-calculus on $L_{\sigma}^{q}\left(\mathbb{R}_{+}^{n}\right)$. This is relevant for further types of functional calculi for linear operators in view of the results in [16].

The strategy for proving the resolvent estimates and the bounded $H^{\infty}$-calculus for the Stokes operator $A_{\alpha}$ is to use the rotation invariance in $n-1$ dimensions of large parts of the constucted solution formula. This enables us to apply the bounded $H^{\infty}$-calculus for the Poisson operator $\left(-\Delta_{\mathbb{R}^{n-1}}\right)^{1 / 2}$ on $L^{q}\left(\mathbb{R}^{n-1}\right)$. In other words we regard the computed representation of the solution as a function of the Poisson operator $g\left(\left(-\Delta_{\mathbb{R}^{n-1}}\right)^{1 / 2}\right)$, which can be estimated in the operator norm on $L^{q}\left(\mathbb{R}^{n-1}\right)$ by the infinity norm of $g$ on a complex sector. Thus, besides the holomorphy of $g$, it suffices to verify pointwise estimates on a complex sector for the terms in the representation of the Stokes resolvent, regarded as functions of $\left(-\Delta_{\mathbb{R}^{n-1}}\right)^{1 / 2}$. This approach seems to be nicer and less complicated than applying multiplier results directly to the lengthy solution formula. The pointwise complex estimates also provide a sufficient decay in the normal component $x_{n}$, such that the $q$-integration over $x_{n}$ afterwards is feasible. By using this metod we first show that the pressure of (1) fullfills $\|\nabla p\|_{q} \leq C\|f\|_{q}$ for some constant $C>0$. Plugging over $\nabla p$ to the right hand side of (1) we then are left with a problem for the Laplacian with data $f-\nabla p$. By well known results for this operator we finally obtain the desired resolvent estimates for the Stokes operator with Robin boundary conditions $A_{\alpha}$ in $L_{\sigma}^{q}\left(\mathbb{R}_{+}^{n}\right)$.

For proving the bounded $H^{\infty}$-calculus for $A_{\alpha}$ we apply the same method. But here we estimate directly the explicit representation for the Stokes flow $u$ of (1).

The local existence result for the Navier-Stokes equations (3) then is a consequence of the proved results for the Stokes operator and the usual fixed point argument. In the situation here we apply directly an abstract result for semilinear parabolic equations in $L^{q}$-spaces of Giga proved in [12].

For the special case of Dirichlet boundary conditions, i.e. in our situation the case $\alpha=$ $\infty$, the above results for Stokes and Navier-Stokes equations are well known. For resolvent estimates see e.g. [26], [18], [28], for maximal regularity see [26], [2], [13], whereas in [7] 
it is proved that the Stokes operator with Dirichlet boundary conditions admits a bounded $H^{\infty}$-calculus on the space $L_{\sigma}^{q}\left(\mathbb{R}_{+}^{n}\right)$ for $1<q<\infty$. On the other hand, as far as the author knows, up to now there are no results available in the literature concerning our type of mixed boundary conditions. We are only aware of some results for various types of other boundary conditions if $\Omega$ is a bounded domain (see e.g. [20], [11], [14]).

We want to remark, that the results presented in this work are included in [24]. There also the case of $L_{\sigma}^{q}\left(\mathbb{R}_{+}^{n}\right)$ with $q=1$ or $q=\infty$ is handled, which will be the content of the forthcoming article [25].

The organization of this work is as follows: After the introduction, in section 2 we will give the notations, used in this work, and will introduce the notion of sectorial operators as well as of operators having a bounded $H^{\infty}$-calculus. Section 3 is dedicated to $L^{p}-L^{q}$ estimates for holomorphic semigroups on $L^{q}$-spaces. In section 4 we construct an explicit representation for the solution of the Stokes resolvent problem with Robin boundary conditions in $\mathbb{R}_{+}^{n}$ which is used to prove the resolvent estimates in section 5 and the bounded $H^{\infty}$-calculus in section 6 for the associated Stokes operator $A_{\alpha}$. Finally, In section 7 the local existence result for the Navier-Stokes equations (3) is stated.

Acknowledgement: The author would like to thank Prof. Dr. Matthias Hieber for attracting his attention to this problem and him and the members of his research group for many fruitful discussions.

\section{Preliminaries}

\section{$2.1 \quad$ Notations}

Here $\Omega$ always denotes an open subset of the real vector space $\mathbb{R}^{n}$. For $m \in\{0,1, \ldots, \infty\}$ we denote by $C^{m}(\Omega)$ the space of all $m$-times continuously differentiable functions and by $C_{c}^{m}(\Omega)$ its subspace consisting of all functions in $C^{m}(\Omega)$ which are compactly supported. Further, let $C_{c}^{m}(\bar{\Omega}):=\left\{\left.u\right|_{\Omega}: u \in C_{c}^{m}\left(\mathbb{R}^{n}\right)\right\}$, and for $m \in \mathbb{N}_{0}:=\mathbb{N} \cup\{0\}$ denote by $C_{b}^{m}(\Omega)$ the Banach space of all $m$-times continuously differentiable functions whose derivatives up to order $m$ are bounded. For the space of all testfunctions we use the abbreviation $\mathcal{D}(\Omega):=C_{c}^{\infty}(\Omega)$ and for its dual $\mathcal{D}^{\prime}(\Omega)$.

The Fourier transform defined on $\mathcal{S}\left(\mathbb{R}^{n}\right)$, the Schwartz space of rapidly decreasing functions, we denote by

$$
\hat{u}(\xi):=\mathcal{F} u(\xi):=\frac{1}{(2 \pi)^{n / 2}} \int_{\mathbb{R}^{n}} \mathrm{e}^{-i x \cdot \xi} u(x) \mathrm{d} x, \quad u \in \mathcal{S}\left(\mathbb{R}^{n}\right) .
$$

For $q \in[1, \infty], L^{q}(\Omega)$ denotes the Lebesgue space, which consists of all $q$-integrable functions if $1 \leq q<\infty$ and $L^{\infty}(\Omega)$ is the space of all functions $u$ that satisfy $\|u\|_{L^{\infty}(\Omega)}:=$

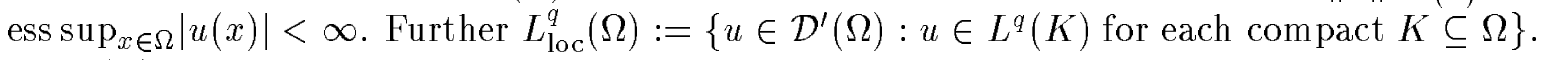
$W^{m, q}(\Omega)$ denotes the Sobolev space of order $m \in \mathbb{N}_{0}$. Its norm is given by

$$
\|u\|_{W^{m, q}(\Omega)}:=\left(\sum_{j=0}^{m}\left\|\nabla^{j} u\right\|_{L^{q}(\Omega)}^{q}\right)^{1 / q}
$$


where $\nabla^{j}$ is the tensor of all possible $j$-th order differentials. Moreover, $W_{0}^{m, q}(\Omega)$ denotes the closure of $C_{c}^{\infty}(\Omega)$ in $W^{m, q}(\Omega)$. If no confusion seems likely, we also write $\|\cdot\|_{q}:=\|\cdot\|_{L^{q}(\Omega)}$ and $\|\cdot\|_{m, q}:=\|\cdot\|_{W^{m, q}(\Omega)}$.

If $\partial \Omega$ is Lipschitz, the trace operator defined by $\gamma(u):=u \int_{\partial \Omega}$ maps $W^{1, q}(\Omega)$ continuously into $W^{1-1 / q, q}(\partial \Omega)$, where

$$
W^{s, q}(\partial \Omega)=\left(L^{q}(\partial \Omega), W^{m, q}(\partial \Omega)\right)_{s / m, q}, 0 \leq s \leq m,
$$

and $(\cdot, \cdot)_{\theta, p}$ denotes the real interpolation space for $0<\theta<1$ and $1 \leq p \leq \infty$. Its kernel is exactly the space $W_{0}^{1, q}(\Omega)$. See [1], p. 215 .

We shall further need the homogeneous Sobolev space $\widehat{W}^{1, q}(\Omega)$ consisting of all $L_{\text {loc }}^{1}(\Omega)$ functions $u$ having finite Dirichlet energy $\int_{\Omega}|\nabla u|^{q} \mathrm{~d} x$, modulo constants. It becomes a Banach space when equipped with the norm

$$
\|u\|_{\widehat{W}^{1, q}(\Omega)}:=\left(\int_{\Omega}|\nabla u|^{q} \mathrm{~d} x\right)^{1 / q} .
$$

Let us remark that we will use the same notation for the corresponding spaces of vector fields on $\Omega$, i.e. $\left(L^{q}(\Omega)\right)^{n}=L^{q}(\Omega),\left(W^{k, q}(\Omega)\right)^{n}=W^{k, q}(\Omega)$, etc. Denote by $q^{\prime}$ the Hölder conjugated exponent, i.e. $\frac{1}{q^{\prime}}+\frac{1}{q}=1$. If $u \in L^{q}(\Omega)$ and $v \in L^{q^{\prime}}(\Omega)$ we use the notation $(u, v):=(u, v)_{\Omega}:=\int_{\Omega} u v \mathrm{~d} x$ for the dual pairing.

If $X$ and $Y$ are Banach spaces, the space of all bounded linear operators from $X$ to $Y$ is denoted by $\mathcal{L}(X, Y)$, and $\mathcal{L}(X)$ is the abbreviation for $\mathcal{L}(X, X)$. For any closed operator $A$ in $X$, its domain and range are denoted by $D(A)$ and $R(A)$, respectively. Its resolvent set is denoted by $\rho(A)$ and its spectrum by $\sigma(A)$. Furthermore, we call $A$ a generator, if $\left(\mathrm{e}^{t A}\right)_{t \geq 0}$ satisfies the semigroup properties.

As usual $C, M, \ldots$ denote constants that may change from line to line. Sometimes we would like to express a special dependence on some parameter $s$. Then we use either the subscript notation $C_{s}, M_{s}, \ldots$ or we write it as an argument $C(s), M(s), \ldots$.

The space of solenoidal fields in the half-space $\mathbb{R}_{+}^{n}$ is defined by $L_{\sigma}^{q}\left(\mathbb{R}_{+}^{n}\right):=\overline{C_{c, \sigma}^{\infty}\left(\mathbb{R}_{+}^{n}\right)}\|\cdot\|_{q}$, where $C_{c, \sigma}^{\infty}\left(\mathbb{R}_{+}^{n}\right):=\left\{v \in C_{c}^{\infty}\left(\mathbb{R}_{+}^{n}\right): \operatorname{div} v=0\right\}$. It is well known that $L_{\sigma}^{q}\left(\mathbb{R}_{+}^{n}\right)=\left\{v \in L^{q}\left(\mathbb{R}_{+}^{n}\right)\right.$ : $\left.\operatorname{div} v=0,\left.v^{n}\right|_{\partial \mathbb{R}_{+}^{n}}=0\right\}$ and that this space is complementary in $L^{q}\left(\mathbb{R}_{+}^{n}\right)$ for $1<q<\infty$. More precisely we have the Helmholtz decomposition

$$
L^{q}\left(\mathbb{R}_{+}^{n}\right)=L_{\sigma}^{q}\left(\mathbb{R}_{+}^{n}\right) \oplus G_{q}\left(\mathbb{R}_{+}^{n}\right),
$$

where $G_{q}\left(\mathbb{R}_{+}^{n}\right):=\left\{\nabla p ; p \in \widehat{W}^{1, q}\left(\mathbb{R}_{+}^{n}\right)\right\}$ (see e.g. [26], [18], [2]). Then the Stokes operator $A_{\alpha, q}$ in $L_{\sigma}^{q}\left(\mathbb{R}_{+}^{n}\right), 1<q<\infty$, is defined by

$$
A_{\alpha, q} u:=-P_{q} \Delta u, \quad u \in D\left(A_{\alpha, q}\right):=\left\{u \in W^{2, q}\left(\mathbb{R}_{+}^{n}\right): T_{\alpha} u=0\right\},
$$

where $P_{q}: L^{q}\left(\mathbb{R}_{+}^{n}\right) \rightarrow L_{\sigma}^{q}\left(\mathbb{R}_{+}^{n}\right)$ is the Helmholtz projection, which is the bounded projection associated to the Helmholtz decomposition. 


\subsection{Sectorial Operators and Bounded $H^{\infty}$-Calculus}

Definition 2.1 A closed operator $A$ in a Banach space $X$ is called sectorial, if it satisfies the following two conditions:

(i) $A$ is densely defined, injective and has dense range,

(ii) $(-\infty, 0) \subseteq \rho(A)$ and there is some $M \geq 0$ such that $\left\|\lambda(\lambda+A)^{-1}\right\| \leq M$ for all $\lambda>0$.

In this case (see e.g. [6]) there is some $\phi \in[0, \pi)$ such that the sector

$$
\Sigma_{\pi-\phi}:=\{z \in \mathbb{C} \backslash\{0\}:|\arg z|<\pi-\phi\}
$$

is contained in $\rho(-A)$, and $\sup \left\{\left|\lambda(\lambda+A)^{-1}\right|: \lambda \in \Sigma_{\pi-\phi}\right\}<\infty$. The infimum over all such $\phi$ is called the spectral angle of $A$ and is denoted by $\phi_{A}$. Oberserve that $\sigma(A) \backslash\{0\} \subset \Sigma_{\phi_{A}}$. Moreover, if $A$ is sectorial, and $\phi_{A} \leq \frac{\pi}{2}$, it generates a bounded holomorphic $C_{0^{-}}$-semigroup on $X$. Recall that $A$ is sectorial if and only if $A^{-1}$ is sectorial and that $D\left(A^{\ell}\right) \cap R\left(A^{\ell}\right)$ is a dense subspace of $X$ for each $\ell \in \mathbb{N}$ (see [6]).

A special class of sectorial operators, on which we will focus in Section 6 , is the set of operators admitting a bounded $H^{\infty}$-calculus. For a comprehensive treatment of this property see [19] and [6]. Before we can introduce operators admitting a bounded $H^{\infty}$-calculus, we need to define for $\phi \in(0, \pi)$ the commutative algebra

$$
H^{\infty}\left(\Sigma_{\phi}\right):=\left\{h: \Sigma_{\phi} \rightarrow \mathbb{C}: h \text { is holomorphic and bounded }\right\}
$$

as well as its subalgebra $H_{0}^{\infty}\left(\Sigma_{\phi}\right)$ given by

$$
H_{0}^{\infty}\left(\Sigma_{\phi}\right):=\left\{h \in H^{\infty}\left(\Sigma_{\phi}\right):|h(z)| \leq C \frac{|z|^{s}}{1+|z|^{2 s}} \text { for some } C \geq 0, s>0\right\} .
$$

Let $A$ be a sectorial operator in $X$ with spectral angle $\phi_{A}$, let $\phi \in\left(\phi_{A}, \pi\right)$ and $\theta \in\left(\phi_{A}, \phi\right)$. The path

$$
\Gamma:=\left\{t \mathrm{e}^{i \theta}: \infty>t>0\right\} \cup\left\{t \mathrm{e}^{-i \theta}: 0 \leq t<\infty\right\},
$$

passing from $\infty \mathrm{e}^{i \theta}$ to $\infty \mathrm{e}^{-i \theta}$, stays in the resolvent set of $A$ with the only possible exception at $t=0$. In view of Cauchy's integral formula, for $h \in H_{0}^{\infty}\left(\Sigma_{\phi}\right)$, we may define $h(A)$ via Dunford's formula by

$$
h(A):=\frac{1}{2 \pi i} \int_{\Gamma} h(\lambda)(\lambda-A)^{-1} \mathrm{~d} \lambda,
$$

which is well defined due to (4) and the sectoriality of $A$. Note that for each sectorial operator $A$, formula (6) defines the algebra homomorphism $\Phi_{A}: H_{0}^{\infty}\left(\Sigma_{\phi}\right) \rightarrow \mathcal{L}(X), \Phi_{A}(h):=h(A)$. The operator $A$ is said to admit a bounded $H^{\infty}$-calculus on $X$, if there is some $C>0$ such that

$$
\|h(A) x\| \leq C\|h\|_{\infty}\|x\|
$$

for all $h \in H_{0}^{\infty}\left(\Sigma_{\phi}\right)$ and all $x \in X$. The infimum over all possible $\phi$ for which inequality (7) holds is called the $H^{\infty}$-angle of $A$ and is denoted by $\phi_{A}^{\infty}$. Clearly, we always have $\phi_{A}^{\infty} \geq \phi_{A}$. We denote by $\mathcal{H}^{\infty}(X)$ the class of all sectorial operators that admit a bounded $H^{\infty}$-calculus on $X$. 
For arbitrary $h \in H^{\infty}\left(\Sigma_{\phi}\right)$ we may define $h(A)$ by the following method. Put $\psi(z)=$ $z(1+z)^{-2}$. Then $\psi \in H_{0}^{\infty}\left(\Sigma_{\phi}\right)$ and by

$$
\frac{\lambda}{(1+\lambda)^{2}}(\lambda-A)^{-1}=-\frac{\lambda}{(1+\lambda)^{2}}(1+A)^{-1}+\frac{1}{1+\lambda}(1+A)^{-2}+(\lambda-A)^{-1} A(1+A)^{-2}
$$

and Cauchy's Theorem we see that $\psi(A)=A(1+A)^{-2}$. Hence this operator is bounded and injective, its range equals $D(A) \cap R(A)$ and its inverse is given by $A^{-1}(1+A)^{2}$. Now for $h \in H^{\infty}\left(\Sigma_{\phi}\right)$ we set

$$
h(A):=(h \psi)(A)[\psi(A)]^{-1}=\frac{1}{2 \pi i}\left(\int_{\Gamma} h(\lambda) \frac{\lambda}{(1+\lambda)^{2}}(\lambda-A)^{-1} \mathrm{~d} \lambda\right)(1+A)^{2} A^{-1},
$$

initially defined on the dense subspace $D(A) \cap R(A)$ of $X$. Thanks to the convergence Lemma (see [5, Lemma 2.1]) it follows that inequality (7) is still valid for those $h$ if $A \in \mathcal{H}^{\infty}(X)$. Consequently, $\Phi_{A}$ extends to bounded algebra homomorphism from $H^{\infty}\left(\Sigma_{\phi}\right)$ to $\mathcal{L}(X)$. Again by identity (8) and Cauchy's Theorem it is easy to see that this definition of $h(A)$ is compatible with the definition (6) in the case $h \in H_{0}^{\infty}\left(\Sigma_{\phi}\right)$.

Since $z \mapsto z^{i s}, s \in[-1,1]$, is a bounded holomorphic function on each sector $\Sigma_{\phi}$, we see that $\mathcal{H}^{\infty}(X)$ is a subclass of

$$
\mathrm{BIP}(X):=\left\{A: D(A) \rightarrow X:\left\|A^{i s}\right\|_{\mathcal{L}(X)} \leq C, s \in[-1,1]\right\} .
$$

So operators in $\mathcal{H}^{\infty}(X)$ enjoy all the properties of this larger class as for instance the representation of the domain of fractional powers $A^{s}$ as a complex interpolation space, i.e. $D\left(A^{s}\right)=[X, D(A)]_{s}, s \in(0,1)$, or the maximal Regularity of the Cauchy problem associated to $A$ if $X$ is a $U M D$ space and $\phi_{A}^{\infty}<\pi / 2$. As mentioned in the introduction, for our purposes in what follows, one important example is the Poisson operator $|\nabla|=\left(-\Delta_{\mathbb{R}^{n}}\right)^{1 / 2}$. Indeed we have $|\nabla| \in \mathcal{H}^{\infty}\left(L^{q}\left(\mathbb{R}^{n}\right)\right)$ for $1<q<\infty$. This follows easily by the selfadjointness of $|\nabla|$ in $L^{2}\left(\mathbb{R}^{n}\right)$, which implies $|\nabla| \in \mathcal{H}^{\infty}\left(L^{2}\left(\mathbb{R}^{n}\right)\right)$, and the results in [8]. Further classes of operators which are known to admit a bounded $H^{\infty}$-calculus are:

Bounded operators, normal sectorial operators in Hilbert spaces (in particular self-adjoint operators) and negative generators of positive contraction semigroups on $L^{q}$-spaces. For details see $[6]$.

\section{$3 \quad L^{p}-L^{q}$-Estimates for Holomorphic Semigroups}

$L^{p}-L^{q}$-estimates for solutions of linear initial value problems have turned out to be an important tool for treating corresponding semilinear equations. For instance in [17] and [12] the authors constructed local strong solutions for the Navier Stokes system by only using such estimates for the solution of the Stokes equations. Here we state $L^{p}-L^{q}$-estimates for certain families of holomorphic $C_{0}$-semigroups on $L^{q}(\Omega)$-spaces. The proof is based on Gagliardo-Nirenbergs inequality as provided in [10], [21] (see also [9, Theorem 9.3]) on $\mathbb{R}^{n}$. In combination with the results in [3] this inequality is also valid for unbounded $(\varepsilon, \infty)$ domains (see also [15]), recalling that for example $\mathbb{R}^{n}, \mathbb{R}_{+}^{n}$ and bent half-spaces belong to this class (see [22, Appendix A] or [24]). 
We state the following results in a more general framework, as in this work it is merely applied to the Stokes semigroup. The expenditure is quite the same. Here let $\left(X_{q}\right)_{q \in(1, \infty)}$ be a family of closed subspaces $X_{q}$ of $L^{q}(\Omega)$ equipped with the $L^{q}$-norm. We assume that

$$
D:=\bigcap_{r \in(1, \infty)} X_{r}
$$

lies dense in each $X_{q}, q \in(1, \infty)$. Furthermore, we suppose $-A_{q}$ to be the generator of a bounded holomorphic $C_{0}$-semigroup $\left(\mathrm{e}^{-t A_{q}}\right)_{t \geq 0}$ on $X_{q}$ and the family $\left(A_{q}\right)_{q \in(1, \infty)}$ to be compatible, i.e. if $u \in D\left(A_{q}\right) \cap D\left(A_{p}\right)$ then $A_{q} u=A_{p} u$ for $p, q \in(1, \infty)$.

Proposition 3.1 Let $\Omega \subseteq \mathbb{R}^{n}$ be an $(\varepsilon, \infty)$ domain. Assume $\left(X_{q}\right)_{q \in(1, \infty)}$ and $\left(A_{q}\right)_{q \in(1, \infty)}$ to be as described above such that

(i) $D\left(A_{q}\right) \hookrightarrow W^{2, q}(\Omega)$ and

(ii) $\left\|\nabla^{2} u\right\|_{q} \leq C\left\|A_{q} u\right\|_{q}, u \in D\left(A_{q}\right)$,

for each $q \in(1, \infty)$. Then there is a constant $C=C(n)$ such that the inequalities

$$
\begin{aligned}
\left\|\mathrm{e}^{-t A_{2}} f\right\|_{p} & \leq C t^{-\frac{n}{2}\left(\frac{1}{q}-\frac{1}{p}\right)}\|f\|_{q}, \quad t>0, f \in X_{q} \cap X_{2}, \\
\left\|\nabla \mathrm{e}^{-t A_{2}} f\right\|_{p} & \leq C t^{-\frac{1}{2}-\frac{n}{2}\left(\frac{1}{q}-\frac{1}{p}\right)}\|f\|_{q}, \quad t>0, f \in X_{q} \cap X_{2},
\end{aligned}
$$

are valid for all $p, q \in(1, \infty]$ satisfying $1<q \leq p<\infty$ or $1<q<p \leq \infty$.

Proof. Let $q \in(1, \infty)$ and $p \in[q, \infty]$. Choosing $q_{1} \in[q, p]$ such that $\frac{1}{q_{1}} \leq \frac{1}{n}+\frac{1}{p}$ yields $a:=\frac{n}{2}\left(\frac{1}{q_{1}}-\frac{1}{p}\right) \leq \frac{1}{2}$. Hence, by assumption and the Gagliardo-Nirenberg inequality, we obtain

$$
\begin{aligned}
\left\|\mathrm{e}^{-t A_{2}} f\right\|_{p} & \leq C\left\|\nabla^{2} \mathrm{e}^{-t A_{q_{1}}} f\right\|_{q_{1}}^{a}\left\|\mathrm{e}^{-t A_{q_{1}}} f\right\|_{q_{1}}^{1-a} \\
& \leq C\left\|A_{q_{1}} \mathrm{e}^{-\frac{t}{2} A_{q_{1}}} \mathrm{e}^{-\frac{t}{2} A_{q_{1}}} f\right\|_{q_{1}}^{a}\left\|\mathrm{e}^{-\frac{t}{2} A_{q_{1}}} \mathrm{e}^{-\frac{t}{2} A_{q_{1}}} f\right\|_{q_{1}}^{1-a} \\
& \leq C_{1} t^{-a}\left\|\mathrm{e}^{-\frac{t}{2} A_{q_{1}}} f\right\|_{q_{1}}
\end{aligned}
$$

for $t>0$ and $f \in D$. Choosing now $q_{2} \in\left[q, q_{1}\right]$ such that $\frac{1}{q_{2}} \leq \frac{1}{n}+\frac{1}{q_{1}} \leq \frac{2}{n}+\frac{1}{p}$ we can continue the above calculation getting

$$
\begin{aligned}
\left\|\mathrm{e}^{-t A_{2}} f\right\|_{p} & \leq C_{1} t^{-\frac{n}{2}\left(\frac{1}{q_{1}}-\frac{1}{p}\right)}\left\|\mathrm{e}^{-\frac{t}{2} A_{q_{1}}} f\right\|_{q_{1}} \\
& \leq C_{2} t^{-\frac{n}{2}\left(\frac{1}{q_{1}}-\frac{1}{p}\right)} t^{-\frac{n}{2}\left(\frac{1}{q_{2}}-\frac{1}{q_{1}}\right)}\left\|\mathrm{e}^{-\frac{t}{4} A_{q_{2}}} f\right\|_{q_{2}} \\
& \leq C_{2} t^{-\frac{n}{2}\left(\frac{1}{q_{2}}-\frac{1}{p}\right)}\left\|\mathrm{e}^{-\frac{t}{4} A_{q_{2}}} f\right\|_{q_{2}} .
\end{aligned}
$$

Iterating this procedure results

$$
\left\|\mathrm{e}^{-t A_{2}} f\right\|_{p} \leq C_{m} t^{-\frac{n}{2}\left(\frac{1}{q_{m}}-\frac{1}{p}\right)}\left\|\mathrm{e}^{-\frac{t}{2^{m}} A_{q_{m}}} f\right\|_{q_{m}}
$$

with $\frac{1}{q_{m}} \leq \frac{m}{n}+\frac{1}{p} \Leftrightarrow q_{m} \geq \frac{n p}{m p+n}$. So, for certain $m \in\{1, \ldots, n\}$, we can reach $q$ and $(10)$ is proved if $f \in D$. 
To see the estimate for the first order derivatives we again apply the Gagliardo-Nirenberg inequality which yields by similar arguments

$$
\begin{aligned}
\left\|\nabla \mathrm{e}^{-t A_{2}} f\right\|_{p} & \leq C\left\|\nabla^{2} \mathrm{e}^{-t A_{q_{1}}} f\right\|_{q_{1}}^{a}\left\|\mathrm{e}^{-t A_{q_{1}}} f\right\|_{q_{1}}^{1-a} \\
& \leq C t^{-a}\left\|\mathrm{e}^{-\frac{t}{2} A_{q_{1}}} f\right\|_{q_{1}}
\end{aligned}
$$

for some $q_{1} \in[2 n, p]$ and $a=\frac{1}{2}+\frac{n}{2}\left(\frac{1}{q_{1}}-\frac{1}{p}\right) \leq \frac{3}{4}$. To continue, if $q \geq 2 n$ we set $q_{1}:=q$, otherwise we use the just proved estimate for $\mathrm{e}^{-t A_{2}}$. In any case we may conclude

$$
\left\|\nabla \mathrm{e}^{-t A_{2}} f\right\|_{p} \leq C t^{-\frac{1}{2}-\frac{n}{2}\left(\frac{1}{q}-\frac{1}{p}\right)}\|f\|_{q}
$$

for $t>0$ and $f \in D$. Since $D$ is assumed to be dense in $X_{q}$ the assertion follows.

If we set $X_{1}:={\overline{D \cap L^{1}(\Omega)}}^{\mid} \cdot \|_{1}$, under some additional assumptions we can extend the above statements to cases where $q=1$.

Proposition 3.2 Let all the assumptions of Proposition 3.1 be fulfilled. Further assume for the families $\left(X_{r}\right)_{r \in[1, \infty)}$ and $\left(A_{r}\right)_{r \in(1, \infty)}$ additionally the properties $X_{r}^{\prime}=X_{r^{\prime}}$ and $A_{r}^{\prime}=A_{r^{\prime}}$, respectively, where $\frac{1}{r}+\frac{1}{r^{\prime}}=1$. Then inequalities (10) and (11) remain true also for values of $p, q \in[1, \infty]$ such that $1 \leq q<p \leq \infty$.

Proof. It remains to verify the two cases $1 \leq q<p<\infty$ and $1=q<p=\infty$. To see the first one, observe that by assumption we have $\left(\mathrm{e}^{-t A_{q}}\right)^{\prime}=\mathrm{e}^{-t A_{q^{\prime}}}$. Thus it follows that

$$
\begin{aligned}
\left\|\mathrm{e}^{-t A_{2}} f\right\|_{p} & =\sup _{v \in D,\|v\|_{p^{\prime}}=1}\left|\left(\mathrm{e}^{-t A_{p}} f, v\right)\right|=\sup _{v \in D,\|v\|_{p^{\prime}}=1}\left|\left(f, \mathrm{e}^{-t A_{p^{\prime}}} v\right)\right| \\
& \leq \sup _{v \in D,\|v\|_{p^{\prime}}=1}\|f\|_{1}\left\|\mathrm{e}^{-t A_{2}} v\right\|_{\infty}
\end{aligned}
$$

for $t>0$ and $f \in D \cap X_{1}$. Applying estimate (10) of Proposition 3.1 we may continue the calculation, which yields

$$
\begin{aligned}
\left\|\mathrm{e}^{-t A_{2}} f\right\|_{p} & \leq C \sup _{v \in D,\|v\|_{p^{\prime}}=1}\|f\|_{1} t^{-\frac{n}{2} \frac{1}{p^{\prime}}}\|v\|_{p^{\prime}} \\
& =C t^{-\frac{n}{2}\left(1-\frac{1}{p}\right)}\|f\|_{1} .
\end{aligned}
$$

This implies for the gradient of $\mathrm{e}^{-t A_{2}} f$

$$
\begin{aligned}
\left\|\nabla \mathrm{e}^{-t A_{2}} f\right\|_{p} & \leq C t^{-\frac{1}{2}}\left\|\mathrm{e}^{-\frac{t}{2} A_{2}} f\right\|_{p} \\
& \leq C t^{-\frac{1}{2}-\frac{n}{2}\left(1-\frac{1}{p}\right)}\|f\|_{1}
\end{aligned}
$$

for $t>0$ and $f \in D \cap X_{1}$, where we applied (11) for the case $1<q=p<\infty$. By density the case $1=q<p<\infty$ is proved.

The case $1=q<p=\infty$ follows by a combination of the cases $1=q<p<\infty$ and $1<q<p=\infty$. 


\section{A Solution Formula for the Resolvent Problem in $\mathbb{R}_{+}^{n}$}

The results for the Stokes operator with Robin boundary conditions, presented in this work, are based on an explicit representation for the resolvent of the Stokes operator in the halfspace $\mathbb{R}_{+}^{n}$, which we state now. For dealing with problems in $\mathbb{R}_{+}^{n}$, the following notation has proved to be useful: $x^{\prime}:=\left(x_{1}, x_{2}, \ldots, x_{n-1}\right) \in \mathbb{R}^{n-1}$ always denotes the first $n-1$ components of the variable $x \in \mathbb{R}_{+}^{n}$, i.e. $x=\left(x^{\prime}, x_{n}\right)=\left(x_{1}, \ldots, x_{n-1}, x_{n}\right)$. We use the same notation for vector fields $u: \mathbb{R}_{+}^{n} \rightarrow \mathbb{C}^{n}$, i.e. $u=\left(u^{\prime}, u^{n}\right)=\left(u^{1}, \ldots, u^{n-1}, u^{n}\right)$ and also for operators, e.g. $R=\left(R^{\prime}, R_{n}\right)=\left(R_{1}, \ldots, R_{n-1}, R_{n}\right)$.

Consider the Stokes resolvent problem

$$
(S R P)_{f, \lambda, \alpha}\left\{\begin{array}{rlll}
(\lambda-\Delta) u+\nabla p & =f & \text { in } \mathbb{R}_{+}^{n}, \\
\operatorname{div} u & =0 & \text { in } \mathbb{R}_{+}^{n}, \\
T_{\alpha} u & =0 & \text { in } \mathbb{R}^{n-1}
\end{array}\right.
$$

where $\lambda \in \Sigma_{\pi-\varphi_{0}}$ for $\varphi_{0} \in(0, \pi)$ and $\alpha \in[0, \infty]$. The right hand side $f$ satisfies the compatibility conditions $\operatorname{div} f=0$ and $f^{n}\left\lceil_{\partial \mathbb{R}_{+}^{n}}=0\right.$, where the trace operator $T_{\alpha}$ is given by

$$
T_{\alpha} u:=\left.\left(\begin{array}{c}
\alpha u^{\prime}-\partial_{n} u^{\prime} \\
u^{n}
\end{array}\right)\right|_{\partial \mathbb{R}_{+}^{n}} .
$$

By a similar way as in [7] we now state a solution formula for $(u, p)$. Fourier transform with respect to the variable $x^{\prime}$ of the system $(S R P)_{f, \lambda, \alpha}$ gives

$$
\begin{aligned}
\left(\lambda+\left|\xi^{\prime}\right|^{2}\right) \hat{u}^{\prime}\left(\xi^{\prime}, x_{n}\right)-\partial_{n}^{2} \hat{u}^{\prime}\left(\xi^{\prime}, x_{n}\right)+i \xi^{\prime} \hat{p}\left(\xi^{\prime}, x_{n}\right) & =\hat{f}^{\prime}\left(\xi^{\prime}, x_{n}\right), \quad \xi^{\prime} \in \mathbb{R}^{n-1}, x_{n}>0, \\
\left(\lambda+\left|\xi^{\prime}\right|^{2}\right) \hat{u}^{n}\left(\xi^{\prime}, x_{n}\right)-\partial_{n}^{2} \hat{u}^{n}\left(\xi^{\prime}, x_{n}\right)+\partial_{n} \hat{p}\left(\xi^{\prime}, x_{n}\right) & =\hat{f}^{n}\left(\xi^{\prime}, x_{n}\right), \quad \xi^{\prime} \in \mathbb{R}^{n-1}, x_{n}>0, \\
i \xi^{\prime} \cdot \hat{u}^{\prime}\left(\xi^{\prime}, x_{n}\right)+\partial_{n} \hat{u}^{n}\left(\xi^{\prime}, x_{n}\right) & =0, \quad \xi^{\prime} \in \mathbb{R}^{n-1}, x_{n}>0 \\
\alpha \hat{u}^{\prime}\left(\xi^{\prime}, 0\right)-\partial_{n} \hat{u}^{\prime}\left(\xi^{\prime}, 0\right) & =0, \quad \xi^{\prime} \in \mathbb{R}^{n-1} \\
\hat{u}^{n}\left(\xi^{\prime}, 0\right) & =0, \quad \xi^{\prime} \in \mathbb{R}^{n-1} .
\end{aligned}
$$

Applying div (in the sense of distributions) to the first line of $(S R P)_{f, \lambda, \alpha}$ we obtain

$$
\Delta p=\operatorname{div} f=0,
$$

where we used $\operatorname{div} u=\operatorname{div} f=0$. This implies that $\hat{p}$ is of the form

$$
\hat{p}\left(\xi^{\prime}, x_{n}\right)=e^{-\left|\xi^{\prime}\right| x_{n}} \hat{p}_{0}\left(\xi^{\prime}\right), \quad \xi^{\prime} \in \mathbb{R}^{n-1}, x_{n}>0,
$$

with some boundary value $\hat{p}_{0}$. We set $\omega\left(\left|\xi^{\prime}\right|\right):=\sqrt{\lambda+\left|\xi^{\prime}\right|^{2}}$ and make the following ansatz for the velocity $u$ :

$$
\begin{aligned}
\hat{u}^{\prime}\left(\xi^{\prime}, x_{n}\right) & =\int_{0}^{\infty} k_{-}\left(\xi^{\prime}, x_{n}, s\right)\left(\hat{f}^{\prime}\left(\xi^{\prime}, s\right)-i \xi^{\prime} \hat{p}\left(\xi^{\prime}, s\right)\right) \mathrm{d} s+e^{-\omega\left(\left|\xi^{\prime}\right|\right) x_{n}} \hat{\phi}^{\prime}\left(\xi^{\prime}\right) \\
\hat{u}^{n}\left(\xi^{\prime}, x_{n}\right) & =\int_{0}^{\infty} k_{+}\left(\xi^{\prime}, x_{n}, s\right)\left(\hat{f}^{n}\left(\xi^{\prime}, s\right)-\partial_{n} \hat{p}\left(\xi^{\prime}, s\right)\right) \mathrm{d} s+e^{-\omega\left(\left|\xi^{\prime}\right|\right) x_{n}} \hat{\phi}^{n}\left(\xi^{\prime}\right)
\end{aligned}
$$

with

$$
k_{ \pm}\left(\xi^{\prime}, x_{n}, s, \lambda\right):=\frac{1}{2 \omega\left(\left|\xi^{\prime}\right|\right)}\left(e^{-\omega\left(\left|\xi^{\prime}\right|\right)\left|x_{n}-s\right|} \pm e^{-\omega\left(\left|\xi^{\prime}\right|\right)\left(x_{n}+s\right)}\right)
$$


and a function $\hat{\phi}=\left(\hat{\phi}^{\prime}, \hat{\phi}^{n}\right)^{T}$ to be determined. For this purpose we use $\operatorname{div} f=0,(18)$ and the identity

$$
\partial_{x_{n}} k_{+}\left(\xi^{\prime}, x_{n}, s\right)=-\partial_{s} k_{-}\left(\xi^{\prime}, x_{n}, s\right)
$$

to obtain

$$
\begin{aligned}
\partial_{n} \hat{u}^{n}\left(\xi^{\prime}, x_{n}\right)= & \int_{0}^{\infty} \partial_{x_{n}} k_{+}\left(\xi^{\prime}, x_{n}, s\right)\left(\hat{f}^{n}\left(\xi^{\prime}, s\right)-\partial_{n} \hat{p}\left(\xi^{\prime}, s\right)\right) \mathrm{d} s-\omega\left(\left|\xi^{\prime}\right|\right) e^{-\omega\left(\left|\xi^{\prime}\right|\right) x_{n}} \hat{\phi}^{n}\left(\xi^{\prime}\right) \\
= & -\int_{0}^{\infty} \partial_{s} k_{-}\left(\xi^{\prime}, x_{n}, s\right)\left(\hat{f}^{n}\left(\xi^{\prime}, s\right)-\partial_{n} \hat{p}\left(\xi^{\prime}, s\right)\right) \mathrm{d} s-\omega\left(\left|\xi^{\prime}\right|\right) e^{-\omega\left(\left|\xi^{\prime}\right|\right) x_{n}} \hat{\phi}^{n}\left(\xi^{\prime}\right) \\
= & -\int_{0}^{\infty} k_{-}\left(\xi^{\prime}, x_{n}, s\right)\left(i \xi^{\prime} \cdot \hat{f}^{\prime}\left(\xi^{\prime}, s\right)+\left|\xi^{\prime}\right|^{2} \hat{p}\left(\xi^{\prime}, s\right)\right) \mathrm{d} s-\omega\left(\left|\xi^{\prime}\right|\right) e^{-\omega\left(\left|\xi^{\prime}\right|\right) x_{n}} \hat{\phi}^{n}\left(\xi^{\prime}\right) \\
= & -i \xi^{\prime} \cdot\left(\int_{0}^{\infty} k_{-}\left(\xi^{\prime}, x_{n}, s\right)\left(\hat{f}^{\prime}\left(\xi^{\prime}, s\right)-i \xi^{\prime} \hat{p}\left(\xi^{\prime}, s\right)\right) \mathrm{d} s+e^{-\omega\left(\left|\xi^{\prime}\right|\right) x_{n}} \hat{\phi}^{\prime}\left(\xi^{\prime}\right)\right) \\
& +e^{-\omega\left(\left|\xi^{\prime}\right|\right) x_{n}}\left(i \xi^{\prime} \cdot \hat{\phi}^{\prime}\left(\xi^{\prime}\right)-\omega\left(\left|\xi^{\prime}\right|\right) \hat{\phi}^{n}\left(\xi^{\prime}\right)\right) \\
= & -i \xi^{\prime} \cdot \hat{u}^{\prime}\left(\xi^{\prime}, x_{n}\right)+e^{-\omega\left(\left|\xi^{\prime}\right|\right) x_{n}}\left(i \xi^{\prime} \cdot \hat{\phi}^{\prime}\left(\xi^{\prime}\right)-\omega\left(\left|\xi^{\prime}\right|\right) \hat{\phi}^{n}\left(\xi^{\prime}\right)\right) .
\end{aligned}
$$

Equality (15) now implies

$$
i \xi^{\prime} \cdot \hat{\phi}^{\prime}\left(\xi^{\prime}\right)=\omega\left(\left|\xi^{\prime}\right|\right) \hat{\phi}^{n}\left(\xi^{\prime}\right), \quad \xi^{\prime} \in \mathbb{R}^{n-1} .
$$

In the following we set

$$
\hat{h}^{n}\left(\xi^{\prime}\right):=\int_{0}^{\infty} e^{-\omega\left(\left|\xi^{\prime}\right|\right) s} \hat{f}^{n}\left(\xi^{\prime}, s\right) \mathrm{d} s, \quad \xi^{\prime} \in \mathbb{R}^{n-1}
$$

and

$$
\hat{h}^{\prime}\left(\xi^{\prime}\right):=\int_{0}^{\infty} e^{-\omega\left(\left|\xi^{\prime}\right|\right) s} \hat{f}^{\prime}\left(\xi^{\prime}, s\right) \mathrm{d} s, \quad \xi^{\prime} \in \mathbb{R}^{n-1} .
$$

Our compatibility assumptions on $f$ then imply for $\xi^{\prime} \in \mathbb{R}^{n-1}$

$$
\begin{aligned}
\omega\left(\left|\xi^{\prime}\right|\right) \hat{h}^{n}\left(\xi^{\prime}\right) & =\int_{0}^{\infty} \omega\left(\left|\xi^{\prime}\right|\right) e^{-\omega\left(\left|\xi^{\prime}\right|\right) s} \hat{f}^{n}\left(\xi^{\prime}, s\right) \mathrm{d} s=-\int_{0}^{\infty} \partial_{s} e^{-\omega\left(\left|\xi^{\prime}\right|\right) s} \hat{f}^{n}\left(\xi^{\prime}, s\right) \mathrm{d} s \\
& =\int_{0}^{\infty} e^{-\omega\left(\left|\xi^{\prime}\right|\right) s} \partial_{s} \hat{f}^{n}\left(\xi^{\prime}, s\right) \mathrm{d} s=-i \xi^{\prime} \cdot \int_{0}^{\infty} e^{-\omega\left(\left|\xi^{\prime}\right|\right) s} \hat{f}^{\prime}\left(\xi^{\prime}, s\right) \mathrm{d} s \\
& =-i \xi^{\prime} \cdot \hat{h}^{\prime}\left(\xi^{\prime}\right) .
\end{aligned}
$$

Further, from (17) and (19) we obtain

$$
\begin{aligned}
0=\hat{u}^{n}\left(\xi^{\prime}, 0\right) & =\frac{1}{\omega\left(\left|\xi^{\prime}\right|\right)} \int_{0}^{\infty} e^{-\omega\left(\left|\xi^{\prime}\right|\right) s}\left(\hat{f}^{n}\left(\xi^{\prime}, s\right)+\left|\xi^{\prime}\right| e^{-\left|\xi^{\prime}\right| s} \hat{p}_{0}\left(\xi^{\prime}\right)\right) \mathrm{d} s+\hat{\phi}^{n}\left(\xi^{\prime}\right) \\
& =\frac{1}{\omega\left(\left|\xi^{\prime}\right|\right)} \hat{h}^{n}\left(\xi^{\prime}\right)+\frac{\left|\xi^{\prime}\right|}{\omega\left(\left|\xi^{\prime}\right|\right)\left(\omega\left(\left|\xi^{\prime}\right|\right)+\left|\xi^{\prime}\right|\right)} \hat{p}_{0}\left(\xi^{\prime}\right)+\hat{\phi}^{n}\left(\xi^{\prime}\right)
\end{aligned}
$$

This equality can be solved with respect to $\hat{p}_{0}$, which leads to

$$
\hat{p}_{0}\left(\xi^{\prime}\right)=-\frac{\omega\left(\left|\xi^{\prime}\right|\right)+\left|\xi^{\prime}\right|}{\left|\xi^{\prime}\right|}\left(\omega\left(\left|\xi^{\prime}\right|\right) \hat{\phi}^{n}\left(\xi^{\prime}\right)+\hat{h}^{n}\left(\xi^{\prime}\right)\right), \quad \xi^{\prime} \in \mathbb{R}^{n-1} .
$$


From the boundary conditions for the remaining components we obtain a second formula for $\hat{p}_{0}$. Using $\left(\partial_{x_{n}} k_{-}\right)\left(\xi^{\prime}, 0, s\right)=e^{-\omega\left(\left|\xi^{\prime}\right|\right) s}$ and (19) a simple calculation yields

$$
\partial_{x_{n}} \hat{u}^{\prime}\left(\xi^{\prime}, 0\right)=\hat{h}^{\prime}\left(\xi^{\prime}\right)-\omega\left(\left|\xi^{\prime}\right|\right) \hat{\phi}^{\prime}\left(\xi^{\prime}\right)-\frac{i \xi^{\prime}}{\omega\left(\left|\xi^{\prime}\right|\right)+\left|\xi^{\prime}\right|} \hat{p}_{0}\left(\xi^{\prime}\right)
$$

Therefore, by (16), it follows

$$
\begin{aligned}
0 & =\alpha \hat{u}^{\prime}\left(\xi^{\prime}, 0\right)-\partial_{n} \hat{u}^{\prime}\left(\xi^{\prime}, 0\right) \\
& =\alpha \hat{\phi}^{\prime}\left(\xi^{\prime}\right)-\left(\hat{h}^{\prime}\left(\xi^{\prime}\right)-\omega\left(\left|\xi^{\prime}\right|\right) \hat{\phi}^{\prime}\left(\xi^{\prime}\right)-\frac{i \xi^{\prime}}{\omega\left(\left|\xi^{\prime}\right|\right)+\left|\xi^{\prime}\right|} \hat{p}_{0}\left(\xi^{\prime}\right)\right)
\end{aligned}
$$

Multiplying this equation with $i \xi^{\prime}$, using (23) and (24) yields for $\xi^{\prime} \in \mathbb{R}^{n-1}$

$$
\hat{p}_{0}\left(\xi^{\prime}\right)=\frac{\omega\left(\left|\xi^{\prime}\right|\right)+\left|\xi^{\prime}\right|}{\left|\xi^{\prime}\right|^{2}} \omega\left(\left|\xi^{\prime}\right|\right)\left[\left(\alpha+\omega\left(\left|\xi^{\prime}\right|\right)\right) \hat{\phi}^{n}\left(\xi^{\prime}\right)+\hat{h}^{n}\left(\xi^{\prime}\right)\right] .
$$

Now, by (25) and (27) $\hat{p}_{0}$ can be eliminated and we obtain the following formula for $\hat{\phi}^{n}$, which depends on the data $f$ of the Stokes resolvent problem only:

$$
\hat{\phi}^{n}\left(\xi^{\prime}\right)=-\frac{\omega\left(\left|\xi^{\prime}\right|\right)+\left|\xi^{\prime}\right|}{\omega\left(\left|\xi^{\prime}\right|\right)+\left|\xi^{\prime}\right|+\alpha} \frac{1}{\omega\left(\left|\xi^{\prime}\right|\right)} \hat{h}^{n}\left(\xi^{\prime}\right), \quad \xi^{\prime} \in \mathbb{R}^{n-1}
$$

A similar calculation as for $\hat{\phi}^{n}$ leads to the following formula for $\hat{\phi}^{\prime}$ :

$$
\hat{\phi}^{\prime}\left(\xi^{\prime}\right)=\frac{1}{\omega\left(\left|\xi^{\prime}\right|\right)+\alpha}\left(\hat{h}^{\prime}\left(\xi^{\prime}\right)+\frac{i \xi^{\prime}}{\left|\xi^{\prime}\right|} \frac{\alpha}{\omega\left(\left|\xi^{\prime}\right|\right)+\left|\xi^{\prime}\right|+\alpha} \hat{h}^{n}\left(\xi^{\prime}\right)\right), \quad \xi^{\prime} \in \mathbb{R}^{n-1} .
$$

\section{Resolvent Estimates for the Stokes Operator}

In the following we prove resolvent estimates for the solution $(u, p)$ of the system $(S R P)_{f, \lambda, \alpha}$ with the help of the formulas constructed in Section 4. The main result in this section reads as follows

Theorem 5.1 Let $n \geq 2,1<q<\infty$, and $\varphi_{0} \in(0, \pi)$. Further let $\alpha \in[0, \infty]$ and $T_{\alpha}$ be defined as in (12). Then for $f \in L^{q}\left(\mathbb{R}_{+}^{n}\right)$ and $\lambda \in \Sigma_{\pi-\varphi_{0}}$ there is a unique solution

$$
(u, p) \in\left\{v \in W^{2, q}\left(\mathbb{R}_{+}^{n}\right): \operatorname{div} v=0, T_{\alpha} v=0\right\} \times \widehat{W}^{1, q}\left(\mathbb{R}_{+}^{n}\right)
$$

of the system $(S R P)_{f, \lambda, \alpha}$.

Moreover, there exists a constant $C=C\left(n, q, \varphi_{0}\right)$, independent of $f, \lambda$, and $\alpha$ such that

$$
|\lambda|\|u\|_{q}+\sqrt{|\lambda|}\|\nabla u\|_{q}+\left\|\nabla^{2} u\right\|_{q}+\|\nabla p\|_{q} \leq C\|f\|_{q} .
$$

Based on this result we verify the existence of a compatible family $\left(A_{\alpha, q}\right)_{q \in(1, \infty)}$ of sectorial operators in $L_{\sigma}^{q}\left(\mathbb{R}_{+}^{n}\right)$ associated to $(S R P)_{f, \lambda, \alpha}$. For proving Theorem 5.1, we first show that $\nabla p \in L^{q}\left(\mathbb{R}_{+}^{n}\right)$. Then, by putting $\nabla p$ on the right hand side, the problem for the Stokes operator is reduced to a resolvent problem for the Laplacian with Robin boundary conditions. 
Hence, the estimates for the solution of that problem, which are also proved below, transfer to the Stokes flow. The proof of Theorem 5.1 requires some further preparations.

To treat the terms appearing in the solution formulas of the just mentioned problems we will frequently make use of the bounded $H^{\infty}$-calculus of the operator

$$
\left|\nabla^{\prime}\right|=\left(-\Delta_{\mathbb{R}^{n-1}}\right)^{1 / 2}=\mathcal{F}^{-1}\left[\xi^{\prime} \mapsto\left|\xi^{\prime}\right|\right] \mathcal{F}
$$

in the space $L^{q}\left(\mathbb{R}^{n-1}\right)$ for $1<q<\infty$. This result provides estimates of the form

$$
\left\|h\left(\left|\nabla^{\prime}\right|\right)\right\|_{\mathcal{L}\left(L^{q}\left(\mathbb{R}^{n-1}\right)\right)} \leq C_{\varphi}\|h\|_{\infty}
$$

for holomorphic functions $h$ that are bounded on the sector $\Sigma_{\varphi}$, where $\varphi \in(0, \pi)$. Since $\phi_{\left|\nabla^{\prime}\right|}^{\infty}=0$, we even may choose $\varphi$ as small as we want. For $f \in \mathcal{S}\left(\mathbb{R}^{n-1}\right)$ and $m \in H_{0}^{\infty}\left(\Sigma_{\varphi}\right)$ we have

$$
\begin{aligned}
\mathcal{F} m\left(\left|\nabla^{\prime}\right|\right) f & =\mathcal{F} \frac{1}{2 \pi i} \int_{\Gamma} m(\lambda)\left(\lambda-\left|\nabla^{\prime}\right|\right)^{-1} f \mathrm{~d} \lambda=\frac{1}{2 \pi i} \int_{\Gamma} m(\lambda) \mathcal{F}\left(\lambda-\left|\nabla^{\prime}\right|\right)^{-1} f \mathrm{~d} \lambda \\
& =\frac{1}{2 \pi i} \int_{\Gamma} m(\lambda)\left(\lambda-\left|\xi^{\prime}\right|\right)^{-1} \hat{f} \mathrm{~d} \lambda=m\left(\left|\xi^{\prime}\right|\right) \hat{f} .
\end{aligned}
$$

By definition (9) it easy follows that the equality above is valid for all $m \in H^{\infty}\left(\Sigma_{\varphi}\right)$. Therefore it remains to show $m \in H^{\infty}\left(\Sigma_{\varphi}\right)$ for some $\varphi \in(0, \pi)$ for proving that $m(|\cdot|)$ is a multiplier on $L^{q}\left(\mathbb{R}^{n-1}\right)$. For this reason the pointwise estimates in the next two lemmas will be very useful in the sequel.

Lemma 5.2 Let $\varphi_{0} \in(0, \pi / 2)$ and $\varphi \in\left(0, \varphi_{0} / 2\right)$. Then there is a constant $C=C\left(\varphi_{0}, \varphi\right)$, such that

(a) $\left|\arg \sqrt{\lambda+z^{2}}\right| \leq \frac{\pi-\varphi_{0}}{2}$,

(b) $\operatorname{Re} \sqrt{\lambda+z^{2}} \geq C \sqrt{|\lambda|}$,

(c) $\operatorname{Re} \sqrt{\lambda+z^{2}} \geq C|z|$

for all $\lambda \in \Sigma_{\pi-\varphi_{0}}$ and $z \in \Sigma_{\varphi}$.

Proof. (a) Since $2 \varphi<\varphi_{0}<\pi / 2<\pi-\varphi_{0}$ we have $\left|\arg \left(\lambda+z^{2}\right)\right| \leq \pi-\varphi_{0}$, hence $\left|\arg \sqrt{\lambda+z^{2}}\right| \leq\left(\pi-\varphi_{0}\right) / 2$.

(b) and (c) In view of $\left|\arg \sqrt{\lambda+z^{2}}\right| \leq\left(\pi-\varphi_{0}\right) / 2<\pi / 2$ the function $f: \Sigma_{\pi-\varphi_{0}} \times \Sigma_{\varphi} \rightarrow \mathbb{R}$, $f(\lambda, z)=\operatorname{Re} \sqrt{\lambda+z^{2}}$ is continuous and strictly positive on the compact set $K:=\{(\lambda, z) \in$ $\left.\bar{\Sigma}_{\pi-\varphi_{0}} \times \bar{\Sigma}_{\varphi}: \sqrt{|\lambda|}+|z|=1\right\}$. This yields $f(\lambda, z) \geq C$ on $K$ for a certain $C>0$, consequently

$$
\begin{aligned}
\operatorname{Re} \sqrt{\lambda+z^{2}} & =\operatorname{Re} \sqrt{\frac{\lambda}{(\sqrt{|\lambda|}+|z|)^{2}}+\left(\frac{z}{(\sqrt{|\lambda|}+|z|)}\right)^{2}}(\sqrt{|\lambda|}+|z|) \\
& \geq C(\sqrt{|\lambda|}+|z|)
\end{aligned}
$$

for all $(\lambda, z) \in \Sigma_{\pi-\varphi_{0}} \times \Sigma_{\varphi}$, which proves (b) and (c). 
We set $\omega(z)=\sqrt{\lambda+z^{2}}$ and fix $\alpha \in[0, \infty]$. Then for $z \in \Sigma_{\varphi}$ and $\lambda \in \Sigma_{\pi-\varphi_{0}}$ we define the functions

$$
\begin{aligned}
G_{\lambda}(z) & :=\frac{z}{\omega(z)} \\
M_{x_{n}, \lambda}(z) & :=\frac{e^{-\omega(z) x_{n}}-e^{-z x_{n}}}{\omega(z)-z}, \\
m_{\lambda}(z) & :=\frac{1}{\omega(z)+z+\alpha} .
\end{aligned}
$$

If we replace $z$ by $\left|\xi^{\prime}\right|$ these are all functions which appear in the solution formulas for the Stokes flow $u$ and the pressure $p$ of of the system $(S R P)_{f, \lambda, \alpha}$.

Lemma 5.3 Let $\sigma, \rho \geq 0, \varphi_{0} \in(0, \pi / 2)$ and $\varphi \in\left(0, \varphi_{0} / 4\right)$. Then there are constants $C, \delta>0$ such that

(a) $\left|G_{\lambda}(z)\right| \leq C$

(b) $\left|z^{1+\rho} M_{x_{n}, \lambda}(z)\right| \leq C \frac{e^{-\delta|z| x_{n}}}{x_{n}^{\rho}\left(1+\sqrt{|\lambda|} x_{n}\right)}$,

(c) $\left|\omega(z) m_{\lambda}(z)\right| \leq C$,

(d) $\left|\alpha m_{\lambda}(z)\right| \leq C \frac{\alpha}{\sqrt{|\lambda|}+\alpha}$,

(e) $\left|z m_{\lambda}(z)\right| \leq C$

(f) $\left|\omega(z)^{\sigma} \mathrm{e}^{-\omega(z) x_{n}}\right| \leq C \frac{\mathrm{e}^{-\delta \sqrt{|\lambda|} x_{n}}}{x_{n}^{\sigma}}$,

for all $z \in \Sigma_{\varphi}, x_{n}>0$ and $\lambda \in \Sigma_{\pi-\varphi_{0}}$.

Proof. (a) From Lemma 5.2 (c) we obtain

$$
\left|\frac{\omega(z)}{z}\right| \geq \operatorname{Re} \sqrt{\frac{\lambda}{|z|^{2}}+\frac{z^{2}}{|z|^{2}}}>C>0, \quad z \in \Sigma_{\varphi}, \quad \lambda \in \Sigma_{\pi-\varphi_{0}}
$$

which shows that (a) holds true.

(b) Let $\lambda \in \Sigma_{\pi-\varphi_{0}}$. For $M_{x_{n}, \lambda}$ we have the representation

$$
M_{x_{n}, \lambda}(z)=-\int_{0}^{x_{n}} e^{-\omega(z)\left(x_{n}-s\right)} e^{-z s} \mathrm{~d} s, \quad z \in \Sigma_{\varphi}, \quad x_{n}>0 .
$$

In virtue of Lemma 5.2 (b) and (c) there is a constant $c_{1}>0$ such that

$$
\operatorname{Re} \omega(z)=\operatorname{Re} \sqrt{\lambda+z^{2}} \geq c_{1}(\sqrt{|\lambda|}+|z|)
$$


for all $z \in \Sigma_{\varphi}$. This implies, if we set $z=r e^{i \theta}$,

$$
\begin{aligned}
\left|z^{1+\rho} M_{x_{n}, \lambda}(z)\right| & =\left|z^{1+\rho} \int_{0}^{x_{n}} e^{-\omega(z)\left(x_{n}-s\right)} e^{-z s} \mathrm{~d} s\right| \\
& \leq|z|^{1+\rho} \int_{0}^{x_{n}}\left|e^{-\omega(z)\left(x_{n}-s\right)}\right|\left|e^{-z s}\right| \mathrm{d} s \\
& \leq r^{1+\rho} \int_{0}^{x_{n}} e^{-\operatorname{Re}(\omega(z))\left(x_{n}-s\right)} e^{-\operatorname{Re}(z) s} \mathrm{~d} s \\
& \leq r^{1+\rho} \int_{0}^{x_{n}} e^{-c_{1}(\sqrt{|\lambda|}+r)\left(x_{n}-s\right)} e^{-r \cos (\theta) s} \mathrm{~d} s .
\end{aligned}
$$

Setting $c_{2}:=\min \left\{c_{1}, \cos (\varphi)\right\}<\cos (\theta)$ we may continue the calculation above obtaining

$$
\begin{aligned}
\left|z^{1+\rho} M_{x_{n}, \lambda}(z)\right| & \leq r^{1+\rho} \int_{0}^{x_{n}} e^{-c_{2}(\sqrt{|\lambda|}+r)\left(x_{n}-s\right)} e^{-c_{2} r s} \mathrm{~d} s \\
& \leq r^{1+\rho} e^{-c_{2} r x_{n}} \int_{0}^{x_{n}} e^{-c_{2} \sqrt{|\lambda|}\left(x_{n}-s\right)} \mathrm{d} s \\
& \leq r^{1+\rho} e^{-c_{2} r x_{n}} \frac{1}{c_{2} \sqrt{|\lambda|}}\left(1-e^{-c_{2} \sqrt{|\lambda|} x_{n}}\right) .
\end{aligned}
$$

The estimate $\sup _{r>0} r^{s} e^{-a r} \leq C \frac{1}{a^{s}}$, which is valid for all $a \in(0, \infty)$ and $s \in[0, \infty)$, now leads to

$$
\begin{aligned}
\left|z^{1+\rho} M_{x_{n}, \lambda}(z)\right| & \leq r^{1+\rho} e^{-c_{2} r x_{n} / 2} e^{-c_{2} r x_{n} / 2} \frac{1}{c_{2} \sqrt{|\lambda|}}\left(1-e^{-c_{2} \sqrt{|\lambda|} x_{n}}\right) \\
& \leq C \frac{e^{-c_{2} r x_{n} / 2}}{x_{n}^{\rho}} \frac{\left(1-e^{-c_{2} \sqrt{|\lambda|} x_{n}}\right)}{c_{2} \sqrt{|\lambda|} x_{n}} \leq C \frac{e^{-c_{2} r x_{n} / 2}}{x_{n}^{\rho}\left(1+c_{2} \sqrt{|\lambda|} x_{n}\right)} \\
& \leq C \frac{e^{-\delta|z| x_{n}}}{x_{n}^{\rho}\left(1+\sqrt{|\lambda|} x_{n}\right)}
\end{aligned}
$$

for $z \in \Sigma_{\varphi}, x_{n}>0$ and $\lambda \in \Sigma_{\pi-\varphi_{0}}$.

(c) According to Lemma 5.2 (a) we have $|\arg \omega(z)| \leq \frac{\pi-\varphi_{0}}{2}$ for $z \in \Sigma_{\varphi}$. This leads to

$$
|-\arg \omega(z)+\arg z| \leq|\arg \omega(z)|+|\arg z| \leq \frac{\pi-\varphi_{0}+2 \varphi}{2}<\frac{\pi}{2}
$$

for $z \in \Sigma_{\varphi}$, since $\varphi \in\left(0, \varphi_{0} / 4\right)$. Hence it follows

$$
\operatorname{Re} \frac{z}{\omega(z)}=\operatorname{Re} \frac{1}{|\omega(z)|} e^{-i \arg \omega(z)}|z| e^{i \arg z}=\frac{|z|}{|\omega(z)|} \cos (-\arg \omega(z)+\arg (z)) \geq 0 .
$$

From $\operatorname{Re} \omega(z) \geq 0$, which is valid according to Lemma $5.2(\mathrm{~b})$, we obtain $\operatorname{Re} \frac{1}{\omega(z)} \geq 0$. Consequently

$$
\operatorname{Re}\left(1+\frac{z}{\omega(z)}+\frac{\alpha}{\omega(z)}\right)=1+\operatorname{Re} \frac{z}{\omega(z)}+\alpha \operatorname{Re} \frac{1}{\omega(z)} \geq 1
$$


which gives

$$
\begin{aligned}
\left|\omega(z) m_{\lambda}(z)\right| & =\left|\frac{\omega(z)}{\omega(z)+z+\alpha}\right| \leq\left|1+\frac{z}{\omega(z)}+\frac{\alpha}{\omega(z)}\right|^{-1} \\
& \leq\left(\operatorname{Re}\left(1+\frac{z}{\omega(z)}+\frac{\alpha}{\omega(z)}\right)\right)^{-1} \leq 1
\end{aligned}
$$

for all $z \in \Sigma_{\varphi}$ and $\lambda \in \Sigma_{\pi-\varphi_{0}}$.

(d) and (e) are analogous to (c).

(f) Here we write $\omega$ as $\omega(z)=r \mathrm{e}^{i \theta}$ with $r>0$ and $\theta \in(0, \varphi)$. By Lemma 5.2 (a) and (b) we get

$$
\begin{aligned}
\mid \omega(z)^{\sigma} \mathrm{e}^{-\omega(z) x_{n} \mid} & =\mid \omega(z)^{\sigma} \mathrm{e}^{-\frac{1}{2} \omega(z) x_{n}} \mathrm{e}^{-\frac{1}{2} \omega(z) x_{n} \mid} \\
& =r^{\sigma} \mathrm{e}^{-\frac{1}{2} r \cos (\theta) x_{n}} \mathrm{e}^{-\frac{1}{2} \operatorname{Re} \omega(z) x_{n}} \leq C \frac{\mathrm{e}^{-\delta \sqrt{|\lambda|} x_{n}}}{\left(\cos (\theta) x_{n}\right)^{\sigma}} \leq C \frac{\mathrm{e}^{-\delta \sqrt{|\lambda|} x_{n}}}{x_{n}^{\sigma}}
\end{aligned}
$$

for $z \in \Sigma_{\varphi}, x_{n}>0$ and $\lambda \in \Sigma_{\pi-\varphi_{0}}$ and the proof is complete.

The next theorem is motivated by our intention to reduce the Stokes system $(S R P)_{f, \lambda, \alpha}$ to a problem for the Laplacian. Consider

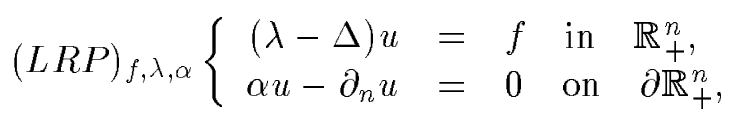

where $\alpha \in[0, \infty]$. The results for the system $(L R P)_{f, \lambda, \alpha}$ stated below are well known. But in order to present a preferably self-contained approach we give a proof by our methods.

Proposition 5.4 Let $n \geq 2, \varphi_{0} \in(0, \pi)$, and $1<q<\infty$. Then for every $f \in L^{q}\left(\mathbb{R}_{+}^{n}\right)$ and $\lambda \in \Sigma_{\pi-\varphi_{0}}$ there is a unique solution $u \in\left\{v \in W^{2, q}\left(\mathbb{R}_{+}^{n}\right): \alpha \gamma v-\gamma \partial_{n} v=0\right\}$ of equation $(L R P)_{f, \lambda, \alpha}$. Further there exists a constant $C=C\left(n, q, \varphi_{0}\right)$, independent of $f, \lambda$, and $\alpha \in$ $[0, \infty]$ such that

$$
|\lambda|\|u\|_{q}+\sqrt{|\lambda|}\|\nabla u\|_{q}+\left\|\nabla^{2} u\right\|_{q} \leq C\|f\|_{q} .
$$

Proof. Since $C_{c}^{\infty}\left(\mathbb{R}_{+}^{n}\right)$ lies dense in $L^{q}\left(\mathbb{R}_{+}^{n}\right)$ we may assume $f \in C_{c}^{\infty}\left(\mathbb{R}_{+}^{n}\right)$. Fourier transform with respect to $x^{\prime}$ yields

$$
(L R P)^{\hat{f}_{, \lambda, \alpha}}\left\{\begin{aligned}
\left(\lambda+\left|\xi^{\prime}\right|^{2}-\partial_{n}^{2}\right) \hat{u}\left(\xi^{\prime}, x_{n}\right) & =\hat{f}\left(\xi^{\prime}, x_{n}\right), \quad \xi^{\prime} \in \mathbb{R}^{n-1}, \quad x_{n} \in \mathbb{R}_{+}, \\
\alpha \hat{u}\left(\xi^{\prime}, 0\right)-\partial_{n} \hat{u}\left(\xi^{\prime}, 0\right) & =0, \quad \xi^{\prime} \in \mathbb{R}^{n-1}
\end{aligned}\right.
$$

Again we set $\omega\left(\left|\xi^{\prime}\right|\right)=\sqrt{\lambda+\left|\xi^{\prime}\right|^{2}}$ and define the extension operators $E^{+}, E^{-}$by

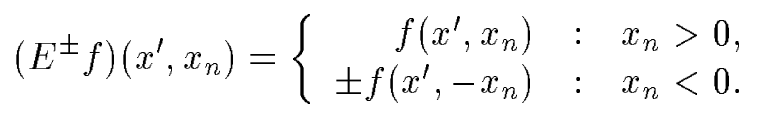

Since the fundamental solution for the operator $\omega^{2}-\partial_{n}^{2}$ is given by

$$
G\left(x_{n}\right)=\frac{1}{2 \omega} e^{-\omega\left|x_{n}\right|}, \quad x_{n} \in \mathbb{R},
$$


we obtain for the convolution with $\mathcal{F} E^{ \pm} f$

$$
\begin{aligned}
\left(G * \mathcal{F} E^{ \pm} f\right)\left\lceil_{\mathbb{R}_{+}^{n}}\left(x_{n}\right)\right. & =\int_{-\infty}^{\infty} \frac{1}{2 \omega} e^{-\omega\left|x_{n}-s\right|} \mathcal{F} E^{ \pm} f(s) \mathrm{d} s \\
& =\int_{0}^{\infty} \frac{1}{2 \omega} e^{-\omega\left|x_{n}-s\right|} \mathcal{F} f(s) \mathrm{d} s+\int_{0}^{\infty} \frac{1}{2 \omega} e^{-\omega\left(x_{n}+s\right)} \mathcal{F} E^{ \pm} f(-s) \mathrm{d} s \\
& =\int_{0}^{\infty} \frac{e^{-\omega\left|x_{n}-s\right|} \pm e^{-\omega\left(x_{n}+s\right)}}{2 \omega} \mathcal{F} f(s) \mathrm{d} s
\end{aligned}
$$

Thus, defining

$$
k_{ \pm}\left(\xi^{\prime}, x_{n}, s, \lambda\right):=\frac{e^{-\omega\left(\left|\xi^{\prime}\right|\right)\left|x_{n}-s\right|} \pm e^{-\omega\left(\left|\xi^{\prime}\right|\right)\left(x_{n}+s\right)}}{2 \omega\left(\left|\xi^{\prime}\right|\right)},
$$

the solution of $(L R P)_{\hat{f}, \lambda, \alpha}$ is of the form

$$
\hat{u}\left(\xi^{\prime}, x_{n}\right)=A_{1} \int_{0}^{\infty} k_{+}\left(\xi^{\prime}, x_{n}, s, \lambda\right) \hat{f}\left(\xi^{\prime}, s\right) \mathrm{d} s+A_{-1} \int_{0}^{\infty} k_{-}\left(\xi^{\prime}, x_{n}, s, \lambda\right) \hat{f}\left(\xi^{\prime}, s\right) \mathrm{d} s,
$$

where $A_{1}$ and $A_{-1}$ are complex valued functions, that may depend on $\xi^{\prime}$. As $\hat{u}$ shall satisfy $\left(\omega(|\cdot|)^{2}-\partial_{n}^{2}\right) \hat{u}=\hat{f}$, the first condition on $A_{1}$ and $A_{-1}$ is

$$
A_{1}+A_{-1}=1 \text {. }
$$

Taking the trace of $\hat{u}$ at $x_{n}=0$ results

$$
\hat{u}\left(\xi^{\prime}, 0\right)=A_{1} \int_{0}^{\infty} \frac{e^{-\omega\left(\left|\xi^{\prime}\right|\right) s}}{\omega\left(\left|\xi^{\prime}\right|\right)} \hat{f}\left(\xi^{\prime}, s\right) \mathrm{d} s=\frac{A_{1}}{\omega\left(\left|\xi^{\prime}\right|\right)} \hat{h}\left(\xi^{\prime}\right),
$$

where $\hat{h}\left(\xi^{\prime}\right):=\int_{0}^{\infty} e^{-\omega\left(\left|\xi^{\prime}\right|\right) s} \hat{f}\left(\xi^{\prime}, s\right) \mathrm{d} s$. By the equality

$$
\partial_{x_{n}} e^{-\omega\left(\left|\xi^{\prime}\right|\right)\left|x_{n}-s\right|}=-\partial_{s} e^{-\omega\left(\left|\xi^{\prime}\right|\right)\left|x_{n}-s\right|}
$$

we obtain

$$
\partial_{x_{n}} k_{ \pm}\left(\xi^{\prime}, x_{n}, s, \lambda\right)=-\partial_{s} k_{\mp}\left(\xi^{\prime}, x_{n}, s, \lambda\right),
$$

which yields

$$
\begin{aligned}
& \partial_{n} \hat{u}\left(\xi^{\prime}, x_{n}\right)= \\
= & -A_{1} \int_{0}^{\infty} \partial_{s} k_{-}\left(\xi^{\prime}, x_{n}, s, \lambda\right) \hat{f}\left(\xi^{\prime}, s\right) \mathrm{d} s-A_{-1} \int_{0}^{\infty} \partial_{s} k_{+}\left(\xi^{\prime}, x_{n}, s, \lambda\right) \hat{f}\left(\xi^{\prime}, s\right) \mathrm{d} s \\
= & A_{1} \int_{0}^{\infty} k_{-}\left(\xi^{\prime}, x_{n}, s, \lambda\right) \partial_{s} \hat{f}\left(\xi^{\prime}, s\right) \mathrm{d} s+A_{-1} \int_{0}^{\infty} k_{+}\left(\xi^{\prime}, x_{n}, s, \lambda\right) \partial_{s} \hat{f}\left(\xi^{\prime}, s\right) \mathrm{d} s .
\end{aligned}
$$

Hence we get for the trace of $\partial_{n} \hat{u}$

$$
\begin{aligned}
\partial_{n} \hat{u}\left(\xi^{\prime}, 0\right) & =A_{-1} \int_{0}^{\infty} \frac{e^{-\omega\left(\left|\xi^{\prime}\right|\right) s}}{\omega\left(\left|\xi^{\prime}\right|\right)} \partial_{s} \hat{f}\left(\xi^{\prime}, s\right) \mathrm{d} s \\
& =-A_{-1} \int_{0}^{\infty} \partial_{s} \frac{e^{-\omega\left(\left|\xi^{\prime}\right|\right) s}}{\omega\left(\left|\xi^{\prime}\right|\right)} \hat{f}\left(\xi^{\prime}, s\right) \mathrm{d} s=A_{-1} \hat{h}\left(\xi^{\prime}\right) .
\end{aligned}
$$


The boundary condition now gives

$$
0=\alpha \gamma \hat{u}-\gamma \partial_{n} \hat{u}=\alpha \frac{A_{1}}{\omega(|\cdot|)} \hat{h}-A_{-1} \hat{h}=\left(\alpha \frac{A_{1}}{\omega(|\cdot|)}-A_{-1}\right) \hat{h} .
$$

Together with the condition $A_{1}+A_{-1}=1$ one obtains

$$
A_{1}\left(\left|\xi^{\prime}\right|\right)=\frac{\omega\left(\left|\xi^{\prime}\right|\right)}{\omega\left(\left|\xi^{\prime}\right|\right)+\alpha} \quad \text { and } \quad A_{-1}\left(\left|\xi^{\prime}\right|\right)=\frac{\alpha}{\omega\left(\left|\xi^{\prime}\right|\right)+\alpha}
$$

for $\xi^{\prime} \in \mathbb{R}^{n-1}$ and $\lambda \in \Sigma_{\pi-\varphi_{0}}$.

Now let $\varphi \in\left(0, \varphi_{0} / 2\right)$. In view of Lemma 5.2 we deduce

$$
\left|A_{-1}(z)\right|=\frac{\alpha}{|\omega(z)+\alpha|} \leq \frac{\alpha}{\operatorname{Re}(\omega(z)+\alpha)} \leq \frac{\alpha}{c \sqrt{|\lambda|}+\alpha} \leq 1
$$

We also have

$$
\left|A_{1}(z)\right|=\frac{1}{\left|1+\frac{\alpha}{\omega(z)}\right|} \leq \frac{1}{1+\alpha \operatorname{Re} \frac{1}{\omega(z)}} \leq 1
$$

for all $z \in \Sigma_{\varphi}, \lambda \in \Sigma_{\pi-\varphi_{0}}$ and $\alpha \in[0, \infty]$. Consequently, $A_{1}\left(\left|\nabla^{\prime}\right|\right)$ and $A_{-1}\left(\left|\nabla^{\prime}\right|\right)$ are bounded operators on $L^{q}\left(\mathbb{R}^{n-1}\right)$ and the operator norm is less then a constant, which does not depend on $\lambda$ and $\alpha$.

Note that

$$
\mathcal{F}^{-1}\left(G * \mathcal{F} E^{ \pm} f\right)\left\lceil_{\mathbb{R}_{+}^{n}}=\left(\lambda-\Delta_{\mathbb{R}^{n}}\right)^{-1} E^{ \pm} f\left\lceil_{\mathbb{R}_{+}^{n}}, \quad \lambda \in \Sigma_{\pi-\varphi_{0}} .\right.\right.
$$

By formula (38) we get the following representation for the solution $u$ :

$$
u=A_{1}\left(\left|\nabla^{\prime}\right|\right)\left(\lambda-\Delta_{\mathbb{R}^{n}}\right)^{-1} E^{+} f\left\lceil\mathbb{R}_{+}^{n}+A_{-1}\left(\left|\nabla^{\prime}\right|\right)\left(\lambda-\Delta_{\mathbb{R}^{n}}\right)^{-1} E^{-} f\left\lceil_{\mathbb{R}_{+}^{n}} .\right.\right.
$$

The assertion now follows because estimate (35) for the resolvent of the Laplacian $\left(\lambda-\Delta_{\mathbb{R}^{n}}\right)^{-1}$ in $L^{q}\left(\mathbb{R}^{n}\right)$ is well known (see $[23]$ ).

Remark 5.5 The above theorem shows that the operator $\Delta_{\alpha}:=\Delta_{\mathbb{R}_{+}^{n}, \alpha}$ defined by $\Delta_{\alpha}:=\Delta$ on $D\left(\Delta_{\alpha}\right):=\left\{v \in W^{2, q}\left(\mathbb{R}_{+}^{n}\right):\left.\left(\alpha v-\partial_{n} v\right)\right|_{\partial \mathbb{R}_{+}^{n}}=0\right\}$ is the generator of a bounded holomorphic

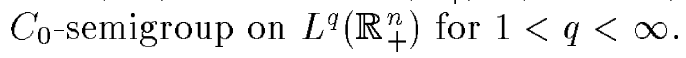

With the above preparations in hands, we turn to the proof of our main result in this section.

\section{Proof. (of Theorem 5.1)}

We prove the estimate

$$
\|\nabla p\|_{L^{q}\left(\mathbb{R}_{+}^{n}\right)} \leq C\|f\|_{L^{q}\left(\mathbb{R}_{+}^{n}\right)}, \quad \lambda \in \Sigma_{\pi-\varphi_{0}} .
$$

To this end, we may assume $f \in L_{\sigma}^{q}\left(\mathbb{R}_{+}^{n}\right)$. Indeed, if $f \in L^{q}\left(\mathbb{R}_{+}^{n}\right)$, then problem $(S R P)_{f, \lambda, \alpha}$ with its solution $(u, p)$ may be rephrased as the problem $(S R P)_{P f, \lambda, \alpha}$, which has the solution $(u, p-q)$, where $\nabla q=(I-P) f$ and $P=P_{\mathbb{R}_{+}^{n}}$ is the Helmholtz projection on $L^{q}\left(\mathbb{R}_{+}^{n}\right)$. So if (41) is proved for $f \in L_{\sigma}^{q}\left(\mathbb{R}_{+}^{n}\right)$, the inequality

$$
\|\nabla p\|_{q} \leq\|\nabla(p-q)\|_{q}+\|\nabla q\|_{q} \leq C\left(\|P f\|_{q}+\|(I-P) f\|_{q}\right) \leq C\|f\|_{q}, \quad \lambda \in \Sigma_{\pi-\varphi_{0}},
$$


implies the estimate for the general case.

For $\xi^{\prime} \in \mathbb{R}^{n-1}$ the term $\partial_{n} \hat{p}$ by (19) can be written as

$$
\begin{aligned}
\partial_{n} \hat{p}\left(\xi^{\prime}, x_{n}\right) & =-\left|\xi^{\prime}\right| e^{-\left|\xi^{\prime}\right| x_{n}} \hat{p}_{0}\left(\xi^{\prime}\right) \\
& =-\left|\xi^{\prime}\right| e^{-\omega\left(\left|\xi^{\prime}\right|\right) x_{n}} \hat{p}_{0}\left(\xi^{\prime}\right)+\left|\xi^{\prime}\right|\left(e^{-\omega\left(\left|\xi^{\prime}\right|\right) x_{n}}-e^{-\left|\xi^{\prime}\right| x_{n}}\right) \hat{p}_{0}\left(\xi^{\prime}\right) .
\end{aligned}
$$

Next we provide a formula for the boundary value $p_{0}$ of the pressure $p$. By (28) and (33) $\hat{\phi}^{n}$ is of the form

$$
\hat{\phi}^{n}\left(\xi^{\prime}\right)=-\left(\omega\left(\left|\xi^{\prime}\right|\right)+\left|\xi^{\prime}\right|\right) m_{\lambda}\left(\left|\xi^{\prime}\right|\right) \frac{1}{\omega\left(\left|\xi^{\prime}\right|\right)} \hat{h}^{n}\left(\xi^{\prime}\right), \quad \xi^{\prime} \in \mathbb{R}^{n-1} .
$$

Hence, in view of $(25)$ we have

$$
\hat{p}_{0}\left(\xi^{\prime}\right)=-\frac{\omega\left(\left|\xi^{\prime}\right|\right)+\left|\xi^{\prime}\right|}{\left|\xi^{\prime}\right|}\left(1-\left(\omega\left(\left|\xi^{\prime}\right|\right)+\left|\xi^{\prime}\right|\right) m_{\lambda}\left(\left|\xi^{\prime}\right|\right)\right) \hat{h}^{n}\left(\xi^{\prime}\right), \quad \xi^{\prime} \in \mathbb{R}^{n-1} .
$$

For the first addend of expression (42) we then deduce

$$
\begin{aligned}
-\left|\xi^{\prime}\right| e^{-\omega\left(\left|\xi^{\prime}\right|\right) x_{n}} \hat{p}_{0}\left(\xi^{\prime}\right) & =e^{-\omega\left(\left|\xi^{\prime}\right|\right) x_{n}}\left(\omega\left(\left|\xi^{\prime}\right|\right)+\left|\xi^{\prime}\right|\right)\left(1-\left(\omega\left(\left|\xi^{\prime}\right|\right)+\left|\xi^{\prime}\right|\right) m_{\lambda}\left(\left|\xi^{\prime}\right|\right)\right) \hat{h}^{n}\left(\xi^{\prime}\right) \\
& =\left(1+G_{\lambda}\left(\left|\xi^{\prime}\right|\right)\right)\left(1-\left(\omega\left(\left|\xi^{\prime}\right|\right)+\left|\xi^{\prime}\right|\right) m_{\lambda}\left(\left|\xi^{\prime}\right|\right)\right) \omega\left(\left|\xi^{\prime}\right|\right) e^{-\omega\left(\left|\xi^{\prime}\right|\right) x_{n}} \hat{h}^{n}\left(\xi^{\prime}\right),
\end{aligned}
$$

whereas inserting $\hat{p}_{0}$ in the second addend of (42) yields

$$
\begin{aligned}
& \left|\xi^{\prime}\right|\left(e^{-\omega\left(\left|\xi^{\prime}\right|\right) x_{n}}-e^{-\left|\xi^{\prime}\right| x_{n}}\right) \hat{p}_{0}\left(\xi^{\prime}\right)= \\
= & -\left|\xi^{\prime}\right| M_{x_{n}, \lambda}\left(\left|\xi^{\prime}\right|\right) \frac{\lambda}{\left|\xi^{\prime}\right|}\left(1-\left(\omega\left(\left|\xi^{\prime}\right|\right)+\left|\xi^{\prime}\right|\right) m_{\lambda}\left(\left|\xi^{\prime}\right|\right)\right) \hat{h}^{n}\left(\xi^{\prime}\right) \\
= & \lambda\left|\xi^{\prime}\right| M_{x_{n}, \lambda}\left(\left|\xi^{\prime}\right|\right)\left(1-\left(\omega\left(\left|\xi^{\prime}\right|\right)+\left|\xi^{\prime}\right|\right) m_{\lambda}\left(\left|\xi^{\prime}\right|\right)\right) \frac{1}{\omega\left(\left|\xi^{\prime}\right|\right)} \frac{i \xi^{\prime}}{\left|\xi^{\prime}\right|} \cdot \hat{h}^{\prime}\left(\xi^{\prime}\right),
\end{aligned}
$$

where we used the abbreviations (31) and (32) and for the last equality (24).

So $\partial_{n} p$ is represented as

$$
\begin{aligned}
\partial_{n} p(x)= & {\left[1-\left(\omega\left(\left|\nabla^{\prime}\right|\right)+\left|\nabla^{\prime}\right|\right) m_{\lambda}\left(\left|\nabla^{\prime}\right|\right)\right]\left(1+G_{\lambda}\left(\left|\nabla^{\prime}\right|\right)\right) \omega\left(\left|\nabla^{\prime}\right|\right) e^{-\omega\left(\left|\nabla^{\prime}\right|\right) x_{n}} \hat{h}^{n}\left(x^{\prime}\right) } \\
& +\left[1-\left(\omega\left(\left|\nabla^{\prime}\right|\right)+\left|\nabla^{\prime}\right|\right) m_{\lambda}\left(\left|\nabla^{\prime}\right|\right)\right] \lambda\left|\nabla^{\prime}\right| M_{x_{n}, \lambda}\left(\left|\nabla^{\prime}\right|\right) \frac{1}{\omega\left(\left|\nabla^{\prime}\right|\right)} R^{\prime} \cdot \hat{h}^{\prime}\left(x^{\prime}\right) .
\end{aligned}
$$

In order to estimate the above formula we need the following lemma.

Lemma 5.6 Let $1<q<\infty$ and $\varphi_{0} \in(0, \pi)$. The components of the function $h$ satisfy

(a) $\left\|h^{j}\right\|_{L^{q\left(\mathbb{R}^{n-1}\right)}} \leq C|\lambda|^{-1 / 2 q^{\prime}}\|f\|_{L^{q}\left(\mathbb{R}_{+}^{n}\right)}, \quad \lambda \in \Sigma_{\pi-\varphi_{0}}, j=1, \ldots, n$,

(b) $\left\|\omega\left(\left|\nabla^{\prime}\right|\right) e^{-\omega\left(\left|\nabla^{\prime}\right|\right)(\cdot) h^{j}}\right\|_{L^{q}\left(\mathbb{R}_{+}^{n}\right)} \leq C\|f\|_{L^{q}\left(\mathbb{R}_{+}^{n}\right)}, \quad \lambda \in \Sigma_{\pi-\varphi_{0}}, j=1, \ldots, n$. 
Proof. We fix $\varphi \in\left(0, \varphi_{0} / 4\right)$. Here we also may use the estimates in Lemma 5.3.

(a) Lemma 5.3 (f) and $\left|\nabla^{\prime}\right| \in \mathcal{H}^{\infty}\left(L^{q}\left(\mathbb{R}^{n-1}\right)\right)$ imply

$$
\begin{aligned}
\left\|h^{j}\right\|_{L^{q}\left(\mathbb{R}^{n-1}\right)} & =\left\|\int_{0}^{\infty} e^{-\omega\left(\left|\nabla^{\prime}\right|\right) s} f^{j}(\cdot, s) \mathrm{d} s\right\|_{L^{q}\left(\mathbb{R}^{n-1}\right)} \\
& \leq \int_{0}^{\infty} e^{-C \sqrt{|\lambda|}}\left\|f^{j}(\cdot, s)\right\|_{L^{q}\left(\mathbb{R}^{n-1}\right)} \mathrm{d} s \\
& \leq\left(\int_{0}^{\infty} e^{-C q^{\prime} \sqrt{|\lambda|} s} \mathrm{~d} s\right)^{1 / q^{\prime}}\left\|f^{j}\right\|_{L^{q}\left(\mathbb{R}_{+}^{n}\right)} \\
& \leq C|\lambda|^{-1 / 2 q^{\prime}}\|f\|_{L^{q}\left(\mathbb{R}_{+}^{n}\right)}
\end{aligned}
$$

for $\lambda \in \Sigma_{\pi-\varphi_{0}}$ and $j=1, \ldots, n$.

(b) Here again Lemma 5.3 (f) leads to

$$
\begin{aligned}
\left\|\omega\left(\left|\nabla^{\prime}\right|\right) e^{-\omega\left(\left|\nabla^{\prime}\right|\right) x_{n}} h^{j}\right\|_{L^{q}\left(\mathbb{R}^{n-1}\right)} & =\left\|\int_{0}^{\infty} \omega\left(\left|\nabla^{\prime}\right|\right) e^{-\omega\left(\left|\nabla^{\prime}\right|\right)\left(x_{n}+s\right)} f^{j}(\cdot, s) \mathrm{d} s\right\|_{L^{q}\left(\mathbb{R}^{n-1}\right)} \\
& \leq C \int_{0}^{\infty} \frac{1}{x_{n}+s}\left\|f^{j}(\cdot, s)\right\|_{L^{q}\left(\mathbb{R}^{n-1}\right)} \mathrm{d} s
\end{aligned}
$$

for $x_{n}>0$. We denote by $\tilde{f}$ the trivial extension of $f$ on $\mathbb{R}^{n}$. By the variable transform $s \mapsto-s$ the latter integral is equal to

$$
\int_{0}^{\infty} \frac{1}{x_{n}+s}\left\|f^{j}(\cdot, s)\right\|_{L^{q}\left(\mathbb{R}^{n-1}\right)} \mathrm{d} s=\int_{-\infty}^{\infty} \frac{1}{x_{n}-s}\left\|\tilde{f}^{j}(\cdot,-s)\right\|_{L^{q}\left(\mathbb{R}^{n-1}\right)} \mathrm{d} s
$$

which is nothing but the Hilbert transform $H$ of the function $x_{n} \mapsto\left\|\tilde{f}^{j}\left(\cdot,-x_{n}\right)\right\|_{L^{q}\left(\mathbb{R}^{n-1}\right)}$ restricted to $\mathbb{R}_{+}$. Since $H$ is bounded on $L^{q}(\mathbb{R})$, there exists a $C>0$ such that

$$
\begin{aligned}
\left\|\omega\left(\left|\nabla^{\prime}\right|\right) e^{-\omega\left(\left|\nabla^{\prime}\right|\right)(\cdot)} h^{j}\right\|_{L^{q}\left(\mathbb{R}_{+}^{n}\right)} & \leq C\left\|H\left(\left\|\tilde{f}^{j}(\cdot,-(\cdot))\right\|_{L^{q}\left(\mathbb{R}^{n-1}\right)}\right)\right\|_{L^{q}\left(\mathbb{R}_{+}\right)} \\
& \leq C\left\|H\left(\left\|\tilde{f}^{j}(\cdot,-(\cdot))\right\|_{L^{q}\left(\mathbb{R}^{n-1}\right)}\right)\right\|_{L^{q}(\mathbb{R})} \\
& \leq C\left\|\tilde{f}^{j}\right\|_{L^{q}\left(\mathbb{R}^{n}\right)} \leq C\|f\|_{L^{q}\left(\mathbb{R}_{+}^{n}\right)}
\end{aligned}
$$

for $\lambda \in \Sigma_{\pi-\varphi_{0}}$ and $j=1, \ldots, n$.

Now, by Lemma 5.3 (a), (b), (c), (e) and since $\left|\nabla^{\prime}\right| \in \mathcal{H}^{\infty}\left(L^{q}\left(\mathbb{R}^{n-1}\right)\right)$ it follows

$$
\begin{aligned}
\left\|\partial_{n} p\right\|_{L^{q}\left(\mathbb{R}_{+}^{n}\right)} \leq & C_{1}\left\|\omega\left(\left|\nabla^{\prime}\right|\right) e^{-\omega\left(\left|\nabla^{\prime}\right|\right)(\cdot)} h^{n}\right\|_{L^{q}\left(\mathbb{R}_{+}^{n}\right)} \\
& +C_{2}\left(\int_{0}^{\infty} \frac{|\lambda|^{q}}{\left(1+\sqrt{|\lambda|} x_{n}\right)^{q}}\left\|\omega\left(\left|\nabla^{\prime}\right|\right)^{-1} R^{\prime} \cdot h^{\prime}\right\|_{L^{q}\left(\mathbb{R}^{n-1}\right)}^{q} \mathrm{~d} x_{n}\right)^{1 / q} .
\end{aligned}
$$

Lemma 5.2 (b), Lemma 5.6 (a) and the boundedness of $R^{\prime}$ in $L^{q}\left(\mathbb{R}^{n-1}\right)$ imply

$$
\begin{aligned}
\left\|\partial_{n} p\right\|_{L^{q}\left(\mathbb{R}_{+}^{n}\right)} & \leq C\left[1+\left(\int_{0}^{\infty} \frac{|\lambda|^{q}}{\left(1+\sqrt{|\lambda|} x_{n}\right)^{q}}|\lambda|^{-\frac{q}{2}\left(1+\frac{1}{q^{\prime}}\right)} \mathrm{d} x_{n}\right)^{1 / q}\right]\|f\|_{L^{q}\left(\mathbb{R}_{+}^{n}\right)} \\
& \leq C\|f\|_{L^{q}\left(\mathbb{R}_{+}^{n}\right)}
\end{aligned}
$$


for $\lambda \in \Sigma_{\pi-\varphi_{0}}$. Since

$$
i \xi^{\prime} \hat{p}\left(\xi^{\prime}, x_{n}\right)=i \xi^{\prime} e^{-\left|\xi^{\prime}\right| x_{n}} \hat{p}_{0}\left(\xi^{\prime}\right)=\frac{i \xi^{\prime}}{\left|\xi^{\prime}\right|}\left|\xi^{\prime}\right| e^{-\left|\xi^{\prime}\right| x_{n}} \hat{p}_{0}\left(\xi^{\prime}\right)=-\frac{i \xi^{\prime}}{\left|\xi^{\prime}\right|} \partial_{n} \hat{p}\left(\xi^{\prime}, x_{n}\right)
$$

for $\xi^{\prime} \in \mathbb{R}^{n-1}$ and $x_{n}>0$, we have $\nabla^{\prime} p=-R^{\prime} \partial_{n} p$. Thus, again by the boundedness of $R^{\prime}$ we also obtain

$$
\left\|\nabla^{\prime} p\right\|_{L^{q}\left(\mathbb{R}_{+}^{n}\right)} \leq C\|f\|_{L^{q}\left(\mathbb{R}_{+}^{n}\right)}, \quad \lambda \in \Sigma_{\pi-\varphi_{0}}
$$

which implies inequality (41).

By putting $\nabla p$ on the right hand side of the Stokes equation we can regard $(S R P)_{f, \lambda, \alpha}$ as the problem $(L R P)_{f-\nabla p, \lambda, \alpha}$ for the Laplacian in $L^{q}\left(\mathbb{R}_{+}^{n}\right)$. Proposition 5.4 then implies together with (41) for the Stokes flow $u$

$$
|\lambda|\|u\|_{q}+\sqrt{|\lambda|}\|\nabla u\|_{q}+\left\|\nabla^{2} u\right\|_{q} \leq C\|f-\nabla p\|_{q} \leq C\left(\|f\|_{q}+\|\nabla p\|_{q}\right) \leq C\|f\|_{q}
$$

for $\lambda \in \Sigma_{\pi-\varphi_{0}}$. Combining this with (41) we deduce estimate (30) and Theorem 5.1 is proved.

Recall that the Stokes operator $A_{\alpha, q}$ in $L_{\sigma}^{q}\left(\mathbb{R}_{+}^{n}\right), 1<q<\infty$, is defined by

$$
A_{\alpha, q} u:=-P_{q} \Delta u, \quad u \in D\left(A_{\alpha, q}\right):=\left\{u \in W^{2, q}\left(\mathbb{R}_{+}^{n}\right): T_{\alpha} u=0\right\},
$$

where $P_{q}$ is the Helmholtz projection on $L^{q}\left(\mathbb{R}_{+}^{n}\right)$. The next theorem shows that $\left(A_{\alpha, q}\right)_{q \in(1, \infty)}$ is a compatible family of sectorial operators in $L_{\sigma}^{q}\left(\mathbb{R}_{+}^{n}\right)$. Essentially, it is a consequence of Theorem 5.1.

Theorem 5.7 Let $1<q<\infty$. The following statements hold true:

(1) $\mathbb{C} \backslash[0, \infty) \subseteq \rho\left(A_{\alpha, q}\right)$ and $\left(\lambda+A_{\alpha, q}\right)^{-1} f=u_{f}(\lambda)$, where $u_{f}(\lambda)$ denotes the unique solution of $(S R P)_{f, \lambda, \alpha}$ for $f \in L_{\sigma}^{q}\left(\mathbb{R}_{+}^{n}\right)$ and $-\lambda \in \rho\left(A_{\alpha, q}\right)$.

(2) $A_{\alpha, q}^{\prime}=A_{\alpha, q^{\prime}}$, where $\frac{1}{q}+\frac{1}{q^{\prime}}=1$. In particular $A_{\alpha, 2}$ is self-adjoint.

(3) $A_{\alpha}:=A_{\alpha, q}$ is sectorial in $L_{\sigma}^{q}\left(\mathbb{R}_{+}^{n}\right)$ with $\phi_{A_{\alpha}}=0$.

Proof. (1) Let $-\lambda \in \mathbb{C} \backslash[0, \infty)$. Then there is a $\varphi_{0} \in(0, \pi)$ such that $\lambda \in \Sigma_{\pi-\varphi_{0}}$. Theorem 5.1 says, that for each $f \in L_{\sigma}^{q}\left(\mathbb{R}_{+}^{n}\right)$ there exists a unique $u=u_{f}(\lambda) \in D\left(A_{\alpha, q}\right)$ such that

$$
\left(\lambda+A_{\alpha, q}\right) u=\left(\lambda-P_{q} \Delta\right) u=P_{q}((\lambda-\Delta) u+\nabla p)=P_{q} f=f
$$

which implies, that $\left(\lambda+A_{\alpha, q}\right): D\left(A_{\alpha, q}\right) \rightarrow L_{\sigma}^{q}\left(\mathbb{R}_{+}^{n}\right)$ is a continuous isomorphism. Thus

$$
\left(\lambda+A_{\alpha, q}\right)^{-1}: L_{\sigma}^{q}\left(\mathbb{R}_{+}^{n}\right) \rightarrow D\left(A_{\alpha, q}\right)
$$

is also a continuous isomorphism and we have $\left(\lambda+A_{\alpha, q}\right)^{-1} f=u_{f}(\lambda)$ for $f \in L_{\sigma}^{q}\left(\mathbb{R}_{+}^{n}\right)$ as well as $-\lambda \in \rho\left(A_{\alpha, q}\right)$. 
(2) Let $u \in D\left(A_{\alpha, q}\right), v \in D\left(A_{\alpha, q^{\prime}}\right)$. By the following calculation we see that

$$
\begin{aligned}
(-\Delta u, v) & =\sum_{j=1}^{n}\left(-\Delta u^{j}, v^{j}\right) \\
& =\sum_{j=1}^{n} \int_{\mathbb{R}^{n-1}} v^{j} \partial_{n} u^{j} \mathrm{~d} x+\sum_{j=1}^{n}\left(\nabla u^{j}, \nabla v^{j}\right) \\
& =\int_{\mathbb{R}^{n-1}} v^{\prime} \cdot \partial_{n} u^{\prime} \mathrm{d} x-\sum_{j=1}^{n} \int_{\mathbb{R}^{n-1}} u^{j} \partial_{n} v^{j} \mathrm{~d} x-\sum_{j=1}^{n}\left(u^{j}, \Delta v^{j}\right) \\
& =\int_{\mathbb{R}^{n-1}} v^{\prime} \cdot \partial_{n} u^{\prime} \mathrm{d} x-\int_{\mathbb{R}^{n-1}} u^{\prime} \cdot \partial_{n} v^{\prime} \mathrm{d} x+(u,-\Delta v) \\
& =-\int_{\mathbb{R}^{n-1}} v^{\prime} \cdot\left(\alpha u^{\prime}-\partial_{n} u^{\prime}\right) \mathrm{d} x+\int_{\mathbb{R}^{n-1}} u^{\prime} \cdot\left(\alpha v^{\prime}-\partial_{n} v^{\prime}\right) \mathrm{d} x+(u,-\Delta v) \\
& =(u,-\Delta v) .
\end{aligned}
$$

This implies

$$
\begin{aligned}
\left(A_{\alpha, q} u, v\right) & =\left(-P_{q} \Delta u, v\right)=\left(-\Delta u, P_{q^{\prime}} v\right)=(-\Delta u, v) \\
& =(u,-\Delta v)=\left(P_{q} u,-\Delta v\right)=\left(u, A_{\alpha, q^{\prime}} v\right)
\end{aligned}
$$

for $u \in D\left(A_{\alpha, q}\right)$ and $v \in D\left(A_{\alpha, q^{\prime}}\right)$. To see that $D\left(A_{\alpha, q}^{\prime}\right) \subseteq D\left(A_{\alpha, q^{\prime}}\right)$ let $v \in D\left(A_{\alpha, q}^{\prime}\right)$ and $w \in\left(L_{\sigma}^{q}\left(\mathbb{R}_{+}^{n}\right)\right)^{\prime}=L_{\sigma}^{q^{\prime}}\left(\mathbb{R}_{+}^{n}\right)$ such that

$$
(u, w)=\left(A_{\alpha, q} u, v\right) \quad \Leftrightarrow \quad(u, w+v)=\left(\left(1+A_{\alpha, q}\right) u, v\right)
$$

for $u \in D\left(A_{\alpha, q}\right)$. According to assertion (1) there is a unique $\tilde{v} \in D\left(A_{\alpha, q^{\prime}}\right)$ such that $\tilde{v}=$ $\left(1+A_{\alpha, q^{\prime}}\right)^{-1}(w+v)$. Now let $f \in L_{\sigma}^{q}\left(\mathbb{R}_{+}^{n}\right)$ and $u=\left(1+A_{\alpha, q}\right)^{-1} f$. Then

$$
\begin{aligned}
(f, v-\tilde{v}) & =\left(\left(1+A_{\alpha, q}\right) u, v\right)-\left(\left(1+A_{\alpha, q}\right) u, \tilde{v}\right) \\
& =(u, v)+(u, w)-(u, w+v)=0 .
\end{aligned}
$$

Hence $v=\tilde{v} \in D\left(A_{\alpha, q^{\prime}}\right)$ and it follows $A_{\alpha, q}^{\prime}=A_{\alpha, q^{\prime}}$.

(3) The statements in (1) show that $A_{\alpha, q}$ is a closed, densely defined operator in $L_{\sigma}^{q}\left(\mathbb{R}_{+}^{n}\right)$ such that, due to $(30)$, for each $\varphi_{0} \in(0, \pi)$ the inequality

$$
\left\|\lambda\left(\lambda+A_{\alpha, q}\right)^{-1}\right\|_{q} \leq C_{\varphi_{0}}, \quad \lambda \in \Sigma_{\varphi_{0}}
$$

is valid for some constant $C_{\varphi_{0}}>0$. Moreover, it is known that a densely defined operator $A: D(A) \rightarrow X$ in a reflexive Banach space $X$ has dense range $R(A)$ if its dual $A^{\prime}$ is injective. In order to obtain (3), therefore it suffices by (2) to show that $A_{\alpha, q}$ is injective for $q \in(1, \infty)$. To this end, let $u \in D\left(A_{\alpha, q}\right)$ satisfying $A_{\alpha, q} u=0$. Since $\left(\lambda+A_{\alpha, q}\right) u=\lambda u$ for $\lambda>0$, we see that $u$ is the solution of the resolvent problem $(S R P)_{f, \lambda, \alpha}$ with $f=\lambda u$. Hence, in view of $(30)$

$$
\left\|\nabla^{2} u\right\|_{q} \leq C\|\lambda u\|_{q}, \quad \lambda>0 .
$$

Letting $\lambda \rightarrow 0$ forces $u$ to be a polynomial of degree less or equal to 1 in $W^{2, q}\left(\mathbb{R}_{+}^{n}\right)$, consequently $u=0$. 
The results in Theorem 5.7 imply in particular that all the assumptions of Proposition 3.1 and Proposition 3.2 for the families $\left(L_{\sigma}^{q}\left(\mathbb{R}_{+}^{n}\right)\right)_{q \in[1, \infty)}$ and $\left(A_{\alpha, q}\right)_{q \in(1, \infty)}$ are fulfilled, if we set $L_{\sigma}^{1}\left(\mathbb{R}_{+}^{n}\right):=\bar{L}^{1}\left(\mathbb{R}_{+}^{n}\right) \cap D{ }^{\|\cdot\|_{1}}$, where $D=\bigcap_{q \in(1, \infty)} L_{\sigma}^{q}\left(\mathbb{R}_{+}^{n}\right)$. Thus the $L^{p}-L^{q}$-estimates stated there are valid for the Stokes semigroup, more precisely we have

Corollary 5.8 The Stokes semigroup $\left(\mathrm{e}^{-t A_{\alpha}}\right)_{t>0}$ satisfies

$$
\begin{aligned}
\left\|\mathrm{e}^{-t A_{\alpha}} f\right\|_{p} & \leq C t^{-\frac{n}{2}\left(\frac{1}{q}-\frac{1}{p}\right)}\|f\|_{q}, \quad t>0, f \in L_{\sigma}^{q}\left(\mathbb{R}_{+}^{n}\right), \\
\left\|\nabla \mathrm{e}^{-t A_{\alpha}} f\right\|_{p} & \leq C t^{-\frac{1}{2}-\frac{n}{2}\left(\frac{1}{q}-\frac{1}{p}\right)}\|f\|_{q}, \quad t>0, f \in L_{\sigma}^{q}\left(\mathbb{R}_{+}^{n}\right),
\end{aligned}
$$

for $p, q \in[1, \infty]$ such that $1<q \leq p<\infty$ or $1 \leq q<p \leq \infty$.

Proof. Since all other assumptions of the Propositions 3.1 and 3.2 follow from the above results, it remains to verify condition (ii) of Proposition 3.1. To this end observe that $u \in$ $D\left(A_{\alpha}\right)$ solves $(S R P)_{f, \lambda, \alpha}$ with right hand side $f=\left(\lambda+A_{\alpha}\right) u$ for $\lambda>0$. Then (30) implies

$$
\left\|\nabla^{2} u\right\|_{q} \leq C\left\|\left(\lambda+A_{\alpha}\right) u\right\|_{q}, \quad \lambda>0
$$

and passing to the limit $\lambda \rightarrow 0$ gives the assertion.

Remark 5.9 By the results in [25] (see also [24]) it follows that (45) is even valid for all values of $p, q \in[1, \infty]$ satisfying $1 \leq q \leq p \leq \infty$, and also (44) except if $p=q=1$ in the case that $\alpha \in(0, \infty]$.

\section{Bounded $H^{\infty}$-Calculus for the Stokes Operator}

In this section we will see that the Stokes operator with Robin boundary conditions admits a bounded $H^{\infty}$-calculus on $L_{\sigma}^{q}\left(\mathbb{R}_{+}^{n}\right)$ for $1<q<\infty$. For the case of Dirichlet boundary conditions this result is already contained in [7]. We start with a lemma and a corollary on integral operators on $L^{q}\left(\mathbb{R}_{+}\right)$, which we copied from [7].

Lemma 6.1 Let $T$ be defined by

$$
(T f)(t)=\int_{0}^{\infty} k(t, s) f(s) \mathrm{d} s, \quad t>0,
$$

where $k: \mathbb{R}_{+} \times \mathbb{R}_{+} \rightarrow \mathbb{C}$ is a measurable function such that the above integral is well defined. Further assume that for some $q \in(1, \infty)$ the operator $T$ satisfies

$$
|(T f)(t)| \leq \frac{C}{t^{1 / q}}\|f\|_{L^{q}\left(\mathbb{R}_{+}\right)}, \quad t>0 .
$$

If $T \in \mathcal{L}\left(L^{q_{0}}\left(\mathbb{R}_{+}\right)\right)$for some $q_{0} \in(q, \infty]$, then $T \in \mathcal{L}\left(L^{p}\left(\mathbb{R}_{+}\right)\right)$for all $p \in\left(q, q_{0}\right]$.

Proof. By assumption $T: L^{q}\left(\mathbb{R}_{+}\right) \rightarrow L_{w}^{q}\left(\mathbb{R}_{+}\right)$is bounded, since $t \mapsto \frac{1}{t^{1 / q}}$ lies in the weak $L^{q}$-space $L_{w}^{q}\left(\mathbb{R}_{+}\right)$(for the notion of weak $L^{q}$-spaces we refer to [27]). The assertion then follows from the Marcinkiewicz interpolation Theorem (see [27, Theorem 2.4.]). 
Corollary 6.2 Let $k: \mathbb{R}_{+} \times \mathbb{R}_{+} \rightarrow \mathbb{C}$ be a measurable function satisfying

$$
|k(t, s)| \leq \frac{C}{t+s} \log \left(1+\frac{t}{s}\right), \quad t, s>0,
$$

and define $T$ as in (46). Then we have $T \in \mathcal{L}\left(L^{p}\left(\mathbb{R}_{+}\right)\right)$for $1<p \leq \infty$.

Proof. Observe that

$$
\int_{0}^{\infty}|k(t, s)| \mathrm{d} s \leq C \int_{0}^{\infty} \frac{\log \left(1+\frac{t}{s}\right)}{1+\frac{t}{s}} \frac{\mathrm{d} s}{s}=C \int_{0}^{\infty} \frac{\log (1+s)}{(1+s) s} \mathrm{~d} s<\infty,
$$

which implies that $T \in \mathcal{L}\left(L^{\infty}\left(\mathbb{R}_{+}\right)\right)$. For $p \in(1, \infty)$ and $f \in L^{p}\left(\mathbb{R}_{+}\right)$we obtain by the Hölder inequality

$$
\begin{aligned}
|(T f)(t)| & \leq C\left(\int_{0}^{\infty} \frac{\left[\log \left(1+\frac{t}{s}\right)\right]^{p^{\prime}} \mathrm{d} s}{(t+s)^{p^{\prime}}}\right)^{1 / p^{\prime}}\|f\|_{L^{p}\left(\mathbb{R}_{+}\right)} \\
& =\frac{C}{t^{1 / p}}\left(\int_{0}^{\infty} \frac{[\log (1+s)]^{p^{\prime}}}{(1+s)^{p^{\prime}}} \frac{\mathrm{d} s}{s^{2-p^{\prime}}}\right)^{1 / p^{\prime}}\|f\|_{L^{p}\left(\mathbb{R}_{+}\right)} \\
& \leq \frac{C}{t^{1 / p}}\|f\|_{L^{p}\left(\mathbb{R}_{+}\right)}
\end{aligned}
$$

for $t>0$. An application of Lemma 6.1 then completes the proof.

Next we state a proper representation for the Stokes flow $u$, which shall be used to prove the bounded $H^{\infty}$-calculus for the Stokes operator. Since $\hat{p}\left(\xi^{\prime}, x_{n}\right)=\mathrm{e}^{-\left|\xi^{\prime}\right| x_{n}} \hat{p}_{0}\left(\xi^{\prime}\right)$, we get by expression (25) for $\hat{p}_{0}$

$$
\begin{aligned}
& \int_{0}^{\infty} k_{+}\left(\xi^{\prime}, x_{n}, s\right) \partial_{n} \hat{p}\left(\xi^{\prime}, s\right) \mathrm{d} s= \\
= & -\int_{0}^{\infty} k_{+}\left(\xi^{\prime}, x_{n}, s\right) \mathrm{e}^{-\left|\xi^{\prime}\right| s} \mathrm{~d} s\left|\xi^{\prime}\right| \hat{p}_{0}\left(\xi^{\prime}\right) \\
= & -\left(\frac{\mathrm{e}^{-\left|\xi^{\prime}\right| x_{n}}-\mathrm{e}^{-\omega\left(\left|\xi^{\prime}\right|\right) x_{n}}}{\omega\left(\left|\xi^{\prime}\right|\right)-\left|\xi^{\prime}\right|}+\frac{\mathrm{e}^{-\omega\left(\left|\xi^{\prime}\right|\right) x_{n}}}{\omega\left(\left|\xi^{\prime}\right|\right)}\right) \frac{\left|\xi^{\prime}\right|}{\omega\left(\left|\xi^{\prime}\right|\right)+\left|\xi^{\prime}\right|} \hat{p}_{0}\left(\xi^{\prime}\right) \\
= & \left(-M_{x_{n}, \lambda}\left(\left|\xi^{\prime}\right|\right)+\frac{\mathrm{e}^{-\omega\left(\left|\xi^{\prime}\right|\right) x_{n}}}{\omega\left(\left|\xi^{\prime}\right|\right)}\right)\left[\omega\left(\left|\xi^{\prime}\right|\right) \hat{\phi}^{n}\left(\xi^{\prime}\right)+\hat{h}^{n}\left(\xi^{\prime}\right)\right]
\end{aligned}
$$

and analogously

$$
\begin{aligned}
\int_{0}^{\infty} k_{-}\left(\xi^{\prime}, x_{n}, s\right) i \xi^{\prime} \hat{p}\left(\xi^{\prime}, s\right) \mathrm{d} s & =\int_{0}^{\infty} k_{-}\left(\xi^{\prime}, x_{n}, s\right) \mathrm{e}^{-\left|\xi^{\prime}\right| s} \mathrm{~d} s i \xi^{\prime} \hat{p}_{0}\left(\xi^{\prime}\right) \\
& =\frac{i \xi^{\prime}}{\left|\xi^{\prime}\right|} M_{x_{n}, \lambda}\left(\left|\xi^{\prime}\right|\right)\left[\omega\left(\left|\xi^{\prime}\right|\right) \hat{\phi}^{n}\left(\xi^{\prime}\right)+\hat{h}^{n}\left(\xi^{\prime}\right)\right] .
\end{aligned}
$$

Inserting this in (21) or (20), respectively, we obtain the representation 


$$
\begin{aligned}
\hat{u}^{n}\left(\xi^{\prime}, x_{n}\right)= & \int_{0}^{\infty} k_{-}\left(\xi^{\prime}, x_{n}, s\right) \hat{f}^{n}\left(\xi^{\prime}, s\right) \mathrm{d} s \\
& +M_{x_{n}, \lambda}\left(\left|\xi^{\prime}\right|\right)\left[\omega\left(\left|\xi^{\prime}\right|\right) \hat{\phi}^{n}\left(\xi^{\prime}\right)+\hat{h}^{n}\left(\xi^{\prime}\right)\right], \quad\left(\xi^{\prime}, x_{n}\right) \in \mathbb{R}_{+}^{n}, \\
\hat{u}^{\prime}\left(\xi^{\prime}, x_{n}\right)= & \int_{0}^{\infty} k_{-}\left(\xi^{\prime}, x_{n}, s\right) \hat{f}^{\prime}\left(\xi^{\prime}, s\right) \mathrm{d} s+e^{-\omega\left(\left|\xi^{\prime}\right|\right) x_{n}} \hat{\phi}^{\prime}\left(\xi^{\prime}\right) \\
& -\frac{i \xi^{\prime}}{\left|\xi^{\prime}\right|} M_{x_{n}, \lambda}\left(\left|\xi^{\prime}\right|\right)\left[\omega\left(\left|\xi^{\prime}\right|\right) \hat{\phi}^{n}\left(\xi^{\prime}\right)+\hat{h}^{n}\left(\xi^{\prime}\right)\right], \quad\left(\xi^{\prime}, x_{n}\right) \in \mathbb{R}_{+}^{n},
\end{aligned}
$$

for the solution $u$ of $(S R P)_{f, \lambda, \alpha}$, where

$$
\hat{\phi}^{n}\left(\xi^{\prime}\right)=-\frac{1}{\omega\left(\left|\xi^{\prime}\right|\right)}\left(\omega\left(\left|\xi^{\prime}\right|\right)+\left|\xi^{\prime}\right|\right) m_{\lambda}\left(\left|\xi^{\prime}\right|\right) \hat{h}^{n}\left(\xi^{\prime}\right), \quad \xi^{\prime} \in \mathbb{R}^{n-1}
$$

and

$$
\hat{\phi}^{\prime}\left(\xi^{\prime}\right)=\frac{1}{\omega\left(\left|\xi^{\prime}\right|\right)+\alpha}\left(\hat{h}^{\prime}\left(\xi^{\prime}\right)+\alpha \frac{i \xi^{\prime}}{\left|\xi^{\prime}\right|} m_{\lambda}\left(\left|\xi^{\prime}\right|\right) \hat{h}^{n}\left(\xi^{\prime}\right)\right), \quad \xi^{\prime} \in \mathbb{R}^{n-1},
$$

according to (28), (29) and (33).

Theorem 6.3 Let $\alpha \in[0, \infty]$. For $1<q<\infty$ the Stokes operator $A_{\alpha}$ admits a bounded $H^{\infty}$-calculus on $L_{\sigma}^{q}\left(\mathbb{R}_{+}^{n}\right)$ with $H^{\infty}$-angle $\phi_{A_{\alpha}}^{\infty}=0$.

Proof. We shall estimate the addends in the formulas (47) and (48) for the Stokes flow $u$ separately. The first addends of $\hat{u}^{n}$ and $\hat{u}^{\prime}$ are transformed representations of the resolvent of the Dirichlet Laplacian $\Delta_{D}$ in $L^{q}\left(\mathbb{R}_{+}^{n}\right)$, which is well known to belong to the class $\mathcal{H}^{\infty}\left(L^{q}\left(\mathbb{R}_{+}^{n}\right)\right)$.

For the second addend of $\hat{u}^{\prime}$ (as former denoted by $\hat{u}_{2}^{\prime}$ ) we have according to (50)

$$
\begin{aligned}
\hat{u}_{2}^{\prime}\left(\xi^{\prime}, x_{n},-\lambda\right)= & \frac{e^{-\omega\left(\left|\xi^{\prime}\right|\right) x_{n}}}{\omega\left(\left|\xi^{\prime}\right|\right)+\alpha}\left(\hat{h}^{\prime}\left(\xi^{\prime}\right)+\alpha \frac{i \xi^{\prime}}{\left|\xi^{\prime}\right|} m_{-\lambda}\left(\left|\xi^{\prime}\right|\right) \hat{h}^{n}\left(\xi^{\prime}\right)\right) \\
= & \int_{0}^{\infty} \frac{e^{-\omega\left(\left|\xi^{\prime}\right|\right)\left(x_{n}+s\right)}}{\omega\left(\left|\xi^{\prime}\right|\right)+\alpha} \hat{f}^{\prime}\left(\xi^{\prime}, s\right) \mathrm{d} s \\
& +\frac{i \xi^{\prime}}{\left|\xi^{\prime}\right|} \int_{0}^{\infty} \alpha \frac{e^{-\omega\left(\left|\xi^{\prime}\right|\right)\left(x_{n}+s\right)}}{\omega\left(\left|\xi^{\prime}\right|\right)+\alpha} m_{-\lambda}\left(\left|\xi^{\prime}\right|\right) \hat{f}^{n}\left(\xi^{\prime}, s\right) \mathrm{d} s
\end{aligned}
$$

for $\left(\xi^{\prime}, x_{n}\right) \in \mathbb{R}_{+}^{n},-\lambda \in \Sigma_{\pi-\varphi_{0}}$ and arbitrary $\varphi_{0} \in(0, \pi / 2)$. Next let $\phi>0, h \in H_{0}^{\infty}\left(\Sigma_{\phi}\right)$ and choose $\varphi_{0} \in(0, \phi)$. We set for $\varphi \in\left(0, \varphi_{0} / 4\right)$

$$
g_{1, \lambda}(z):=\frac{e^{-\omega(z)\left(x_{n}+s\right)}}{\omega(z)+\alpha}, \quad z \in \Sigma_{\varphi}
$$

and

$$
g_{2, \lambda}(z):=\alpha m_{-\lambda}(z) \frac{e^{-\omega(z)\left(x_{n}+s\right)}}{\omega(z)+\alpha}, \quad z \in \Sigma_{\varphi},
$$

and consider the functions

$$
G_{j}(z):=\frac{1}{2 \pi i} \int_{\Gamma} h(\lambda) g_{j, \lambda}(z) \mathrm{d} \lambda, \quad z \in \Sigma_{\varphi}, j=1,2,
$$




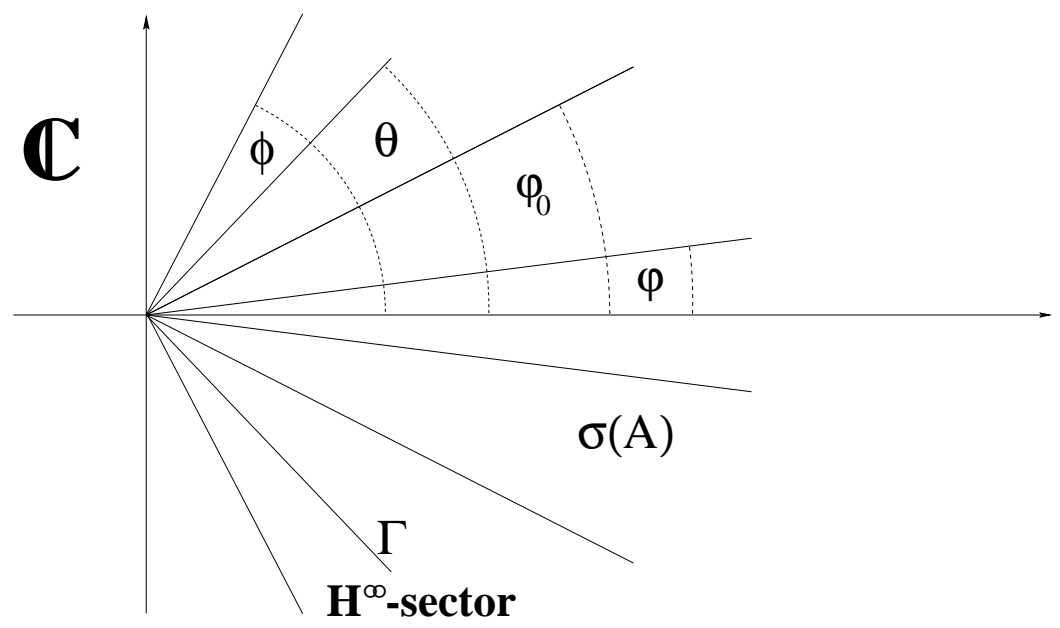

Figure 1: The angles $\varphi, \varphi_{0}, \theta, \phi$.

where $\Gamma:=\left\{\mu \in \mathbb{C}: \mu=r \mathrm{e}^{i \theta}, r \geq 0\right\}$ for some $\theta \in\left(\varphi_{0}, \phi\right)$ (see figure 1 for a sketch of the different angles used in this proof). In virtue of Lemma 5.2 (b) and Lemma 5.3 (d), (f), the functions $G_{j}, j=1,2$, can be estimated by

$$
\begin{aligned}
\left|G_{j}(z)\right| & \leq C\|h\|_{\infty} \int_{0}^{\infty} \frac{\mathrm{e}^{-c_{1} \sqrt{\sigma}\left(x_{n}+s\right)}}{\sqrt{\sigma}} \mathrm{d} \sigma \\
& =C\|h\|_{\infty} \int_{0}^{\infty} \mathrm{e}^{-c_{1} \tilde{\sigma}\left(x_{n}+s\right)} \mathrm{d} \tilde{\sigma} \\
& \leq \frac{C}{x_{n}+s}\|h\|_{\infty}, \quad z \in \Sigma_{\varphi}, x_{n}, s>0,
\end{aligned}
$$

for all $\alpha \in[0, \infty]$. Thus $G_{j} \in H^{\infty}\left(\Sigma_{\varphi}\right)$ for $j=1,2$ and we obtain by the bounded $H^{\infty}$-calculus for the operator $\left|\nabla^{\prime}\right|$ on $L^{q}\left(\mathbb{R}^{n-1}\right)$

$$
\begin{aligned}
& \left\|\frac{1}{2 \pi i} \int_{\Gamma} h(\lambda) u_{2}^{\prime}\left(\cdot, x_{n},-\lambda\right) \mathrm{d} \lambda\right\|_{L^{q}\left(\mathbb{R}^{n-1}\right)} \leq \\
\leq & \left\|\int_{0}^{\infty} G_{1}\left(\left|\nabla^{\prime}\right|\right) f^{\prime}(\cdot, s) \mathrm{d} s\right\|_{L^{q}\left(\mathbb{R}^{n-1}\right)}+\left\|R^{\prime} \int_{0}^{\infty} G_{2}\left(\left|\nabla^{\prime}\right|\right) f^{n}(\cdot, s) \mathrm{d} s\right\|_{L^{q}\left(\mathbb{R}^{n-1}\right)} \\
\leq & C\|h\|_{\infty} \int_{0}^{\infty} \frac{\|f(\cdot, s)\|_{L^{q\left(\mathbb{R}^{n-1}\right)}}}{x_{n}+s} \mathrm{~d} s \\
= & C\|h\|_{\infty} \int_{-\infty}^{0} \frac{\|f(\cdot,-s)\|_{L^{q\left(\mathbb{R}^{n-1}\right)}}}{x_{n}-s} \mathrm{~d} s
\end{aligned}
$$

for $x_{n}>0$. Setting $\tilde{f}\left(x^{\prime},-s\right):=\left\{\begin{array}{r}f\left(x^{\prime},-s\right) \quad: \quad s<0, \\ 0 \quad: \quad s \geq 0,\end{array}\right.$ and using the boundedness of the Hilbert transform $H$ on $L^{q}(\mathbb{R})$ we obtain

$$
\begin{aligned}
\left\|\frac{1}{2 \pi i} \int_{\Gamma} h(\lambda) u_{2}^{\prime}(\cdot, \cdot,-\lambda) \mathrm{d} \lambda\right\|_{L^{q}\left(\mathbb{R}_{+}^{n}\right)} & \leq C\|h\|_{\infty}\left\|H\left(\|\tilde{f}(\cdot,-\cdot)\|_{L^{q}\left(\mathbb{R}^{n-1}\right)}\right)\right\|_{L^{q}(\mathbb{R})} \\
& \leq C\|h\|_{\infty}\|\tilde{f}\|_{L^{q}\left(\mathbb{R}^{n}\right)} \\
& \leq C\|h\|_{\infty}\|f\|_{L^{q\left(\mathbb{R}_{+}^{n}\right)}} .
\end{aligned}
$$


By (49) the second term of $\hat{u}^{n}$ can be written as

$$
\begin{aligned}
& \hat{u}_{2}^{n}\left(\xi^{\prime}, x_{n},-\lambda\right)= \\
= & M_{x_{n},-\lambda}\left(\left|\xi^{\prime}\right|\right)\left[1-\left(\omega\left(\left|\xi^{\prime}\right|\right)+\left|\xi^{\prime}\right|\right) m_{-\lambda}\left(\left|\xi^{\prime}\right|\right)\right] \hat{h}^{n}\left(\xi^{\prime}\right) \\
= & \frac{i \xi^{\prime}}{\left|\xi^{\prime}\right|} \cdot \int_{0}^{\infty} \frac{\left|\xi^{\prime}\right|}{\omega\left(\left|\xi^{\prime}\right|\right)} M_{x_{n},-\lambda}\left(\left|\xi^{\prime}\right|\right)\left[1-\left(\omega\left(\left|\xi^{\prime}\right|\right)+\left|\xi^{\prime}\right|\right) m_{-\lambda}\left(\left|\xi^{\prime}\right|\right)\right] e^{-\omega\left(\left|\xi^{\prime}\right|\right) s} \hat{f}^{\prime}\left(\xi^{\prime}, s\right) \mathrm{d} s
\end{aligned}
$$

for $\left(\xi^{\prime}, x_{n}\right) \in \mathbb{R}_{+}^{n}$ and $-\lambda \in \Sigma_{\pi-\varphi_{0}}$. This time we set

$$
\begin{aligned}
g_{\lambda}(z) & :=\frac{z}{\omega(z)} M_{x_{n},-\lambda}(z)\left[1-(\omega(z)+z) m_{-\lambda}(z)\right] e^{-\omega(z) s} \\
& =-\frac{z}{\omega(z)} \int_{0}^{x_{n}} \mathrm{e}^{-\omega(z)\left(x_{n}-\rho+s\right)} \mathrm{e}^{-z \rho}\left[1-(\omega(z)+z) m_{-\lambda}(z)\right] \mathrm{d} \rho, \quad z \in \Sigma_{\varphi}
\end{aligned}
$$

and deduce with $r=|z|$ by Lemma 5.2 and Lemma 5.3

$$
\begin{aligned}
|G(z)| & =\left|\frac{1}{2 \pi i} \int_{\Gamma} h(\lambda) g_{\lambda}(z) \mathrm{d} \lambda\right| \\
& \leq C\|h\|_{\infty} \int_{0}^{\infty} \frac{r}{r+\sqrt{\sigma}} \int_{0}^{x_{n}} \mathrm{e}^{-c_{1}(r+\sqrt{\sigma})\left(x_{n}-\rho+s\right)} \mathrm{e}^{-c_{1} r \rho} \mathrm{d} \rho \mathrm{d} \sigma \\
& \leq C\|h\|_{\infty} r \mathrm{e}^{-c_{1} r\left(x_{n}+s\right)} \int_{0}^{x_{n}} \int_{0}^{\infty} \frac{1}{r+\sqrt{\sigma}} \mathrm{e}^{-c_{1} \sqrt{\sigma}\left(x_{n}-\rho+s\right)} \mathrm{d} \sigma \mathrm{d} \rho .
\end{aligned}
$$

Here we applied Lemma 5.2 (b) and (c) to $\mathrm{e}^{-\omega(z)\left(x_{n}-\rho+s\right)}$. Since $\sup _{r>0} r \mathrm{e}^{-c_{1} r\left(x_{n}+s\right)} \leq \frac{C}{x_{n}+s}$ we can continue the calculation by the substitution $\sigma \mapsto \sqrt{\sigma}$ and obtain

$$
\begin{aligned}
|G(z)| & \leq C\|h\|_{\infty} \frac{1}{\left(x_{n}+s\right)} \int_{0}^{x_{n}} \int_{0}^{\infty} \frac{\sigma}{r+\sigma} \mathrm{e}^{-c_{1} \sigma\left(x_{n}-\rho+s\right)} \mathrm{d} \sigma \mathrm{d} \rho \\
& \leq C\|h\|_{\infty} \frac{1}{\left(x_{n}+s\right)} \int_{0}^{x_{n}} \frac{1}{\left(x_{n}-\rho+s\right)} \mathrm{d} \rho \\
& =C\|h\|_{\infty} \frac{1}{\left(x_{n}+s\right)} \log \left(1+\frac{x_{n}}{s}\right), \quad x_{n}, s>0 .
\end{aligned}
$$

This implies

$$
\begin{aligned}
\left\|\frac{1}{2 \pi i} \int_{\Gamma} h(\lambda) u_{2}^{n}\left(\cdot, x_{n},-\lambda\right) \mathrm{d} \lambda\right\|_{L^{q\left(\mathbb{R}^{n-1}\right)}} & \leq\left\|R^{\prime} \cdot \int_{0}^{\infty} G\left(\left|\nabla^{\prime}\right|\right) f^{\prime}(\cdot, s) \mathrm{d} s\right\|_{L^{q}\left(\mathbb{R}^{n-1}\right)} \\
& \leq C\|h\|_{\infty} \int_{0}^{\infty} \frac{\log \left(1+\frac{x_{n}}{s}\right)}{x_{n}+s}\|f(\cdot, s)\|_{L^{q\left(\mathbb{R}^{n-1}\right)}} \mathrm{d} s
\end{aligned}
$$

for $x_{n}>0$. Corollary 6.2 then yields

$$
\left\|\frac{1}{2 \pi i} \int_{\Gamma} h(\lambda) u_{2}^{n}\left(\cdot, x_{n},-\lambda\right) \mathrm{d} \lambda\right\|_{L^{q}\left(\mathbb{R}_{+}^{n}\right)} \leq C\|h\|_{\infty}\|f\|_{L^{q}\left(\mathbb{R}_{+}^{n}\right)}
$$

and, since $u_{3}^{\prime}=-R^{\prime} u_{2}^{n}$, the same estimate is valid for the third addend of $u^{\prime}$.

Summarizing and having in mind that $(\lambda-A)^{-1} f=-u(\cdot,-\lambda)$ for $-\lambda \in \Sigma_{\pi-\varphi_{0}}$ we may conclude that

$$
\|h(A) f\|_{L^{q\left(\mathbb{R}_{+}^{n}\right)}}=\left\|\frac{1}{2 \pi i} \int_{\Gamma} h(\lambda)(\lambda-A)^{-1} f \mathrm{~d} \lambda\right\|_{L^{q\left(\mathbb{R}_{+}^{n}\right)}} \leq C\|h\|_{\infty}\|f\|_{L^{q}\left(\mathbb{R}_{+}^{n}\right)}
$$

for all $h \in H_{0}^{\infty}\left(\Sigma_{\phi}\right), f \in L_{\sigma}^{q}\left(\mathbb{R}_{+}^{n}\right)$ and the assertion follows. 


\section{A Local Existence Result for the Navier-Stokes Equations}

To prove a local existence result for problem (3) we intend to apply Theorem 1 in [12]. In an abstract setting this result provides a unique local solution for semilinear parabolic initial value problems of the form

$$
\left\{\begin{aligned}
u^{\prime}+A u+F(u) & =0 \quad \text { in } \quad(0, T), \\
u(0) & =u_{0}
\end{aligned}\right.
$$

where $u_{0}$ belongs to a certain $L^{q}$-space $E^{q}, A$ is a generator of a $C_{0^{-}}$-semigroup, and $F$ is the nonlinear part. In particular Theorem 1 in [12] states the existence of a mild solution of (51), i.e. a solution of the corresponding integral equation

$$
u(t)=\mathrm{e}^{-A t} u_{0}+\int_{0}^{\infty} \mathrm{e}^{-(t-s) A} F(u(s)) \mathrm{d} s, \quad t \in(0, T) .
$$

In our situation, by applying the Helmholtz projection $P=P_{\mathbb{R}_{+}^{n}}$ to (3), we see that this equation can be written as a problem of the form (51) with $E^{q}=L_{\sigma}^{q}\left(\mathbb{R}_{+}^{n}\right), F(u)=P(u, \nabla) u$, and $A=A_{\alpha}=A_{\alpha, q}$ the Stokes operator with Robin boundary conditions in $L_{\sigma}^{q}\left(\mathbb{R}_{+}^{n}\right)$.

By checking the assumptions for the abstract existence result in [12] one will realize that the main work for applying Theorem 1 is done in the previous sections. For instance the $L^{p}-L^{q}$-estimates in (10) equal exactly assumption (A) in [12] (with $m=2$ ). Since div $u=0$ we may write the nonlinearity $F$ as $F=\sum_{j=1}^{n} \Gamma_{j} G_{j}$, where $\Gamma_{j} v:=P \partial_{j} v$ and $G(v):=v^{j} v$. Since $\left(A_{\alpha, q}\right)^{\prime}=A_{\alpha, q^{\prime}}$ by a duality argument and in view of estimate (11) we easy see that

$$
\left\|\mathrm{e}^{-t A} \Gamma_{j} v\right\|_{q} \leq C t^{-1 / 2}\|v\|_{q}, \quad 0<t<\infty, 1<q<\infty, v \in L_{\sigma}^{q}\left(\mathbb{R}_{+}^{n}\right) .
$$

Hence, also assumption (N1) in [12] is satisfied (with $\gamma=1$ ). For the nonlinearities $G_{j}$ we obtain by applying the Hölder inequality

$$
\begin{aligned}
\left\|G_{j} v-G_{j} w\right\|_{q / 2} & \leq C\left(\left\|\left(v_{j}-w_{j}\right) v\right\|_{q / 2}+\left\|w_{j}(v-w)\right\|_{q / 2}\right) \\
& \leq C\|v-w\|_{q}\left(\|v\|_{q}+\|w\|_{q}\right)
\end{aligned}
$$

for $1<q<\infty, v, w \in L_{\sigma}^{q}\left(\mathbb{R}_{+}^{n}\right)$. This implies that also the third and last assumption (N2) of Theorem 1 in [12] is fullfilled (with $\alpha=1$ ). Thus, we may apply this result to our situation and we deduce

Theorem 7.1 Let $n \leq q<\infty$. Then for each $u_{0} \in L_{\sigma}^{q}\left(\mathbb{R}_{+}^{n}\right)$ there is a $T_{0}>0$ and a unique mild solution of (3) on $\left[0, T_{0}\right)$ such that

(1) $u \in \mathrm{BC}\left(\left[0, T_{0}\right) ; L_{\sigma}^{q}\left(\mathbb{R}_{+}^{n}\right)\right) \cap L^{r}\left(\left(0, T_{0}\right) ; L_{\sigma}^{q}\left(\mathbb{R}_{+}^{n}\right)\right)$,

(2) $t^{1 / r} u \in \mathrm{BC}\left(\left[0, T_{0}\right) ; L_{\sigma}^{s}\left(\mathbb{R}_{+}^{n}\right)\right)$ with $t^{1 / r}\|u(t)\|_{q} \rightarrow 0$ if $t \rightarrow 0$

for $\frac{2}{r}+\frac{n}{s}=\frac{n}{q}, r, s>q$.

(3) Let $q=n>1$. There is an $\varepsilon>0$ such that $T_{0}$ can be chosen as $\infty$ if $\left\|u_{0}\right\|_{q}<\varepsilon$, and we have

$$
\|u(t)\|_{s} \leq C t^{-\frac{n}{2}\left(\frac{1}{q}-\frac{1}{s}\right)}, \quad 0<t<\infty,
$$

if $s \geq q$. 
(4) If $q>n>1$ then

$$
T_{0} \geq C\left\|u_{0}\right\|_{q}^{-\frac{n}{2}\left(\frac{1}{n}-\frac{1}{q}\right)} .
$$

(5) If $q>n>1$ and $\left(0, T^{*}\right)$ is the maximal existence interval of the solution $u$ in $C\left(\left(0, T^{*}\right) ; L_{\sigma}^{q}\left(\mathbb{R}_{+}^{n}\right)\right)$ then

$$
\|u(t)\|_{q} \geq \frac{C}{\left(T^{*}-t\right)^{(q-n) / 2 q}}, \quad t \in\left(0, T^{*}\right) .
$$

\section{References}

[1] R.A. Adams. Sobolev Spaces. Academic Press, 1978.

[2] W. Borchers and T. Miyakawa. $L^{2}$ decay for the Navier-Stokes flow in halfspaces. Math. Ann., 282:139-155, 1988.

[3] S.-K. Chua. Extension theorems on weighted Sobolev spaces. Indiana Univ. Math. J., 41:1027-1076, 1992 .

[4] P. Clément, B. de Pagter, F.A. Sukochev, and H. Witvliet. Schauder decompositions and multiplier theorems. Studia Math., 138:135-163, 2000.

[5] M. Cowling, I. Doust, A. McIntosh, and A. Yagi. Banach space operators with a bounded $H^{\infty}$ functional calculus. J. Austral. Math. Soc., 60:51-89, 1996.

[6] R. Denk, M. Hieber, and J. Prüss. R-boundedness, Fourier multipliers and problems of elliptic and parabolic type. Mem. Amer. Math. Soc., 166:viii+114, 2003.

[7] W. Desch, M. Hieber, and J. Prüss. $L^{p}$-theory of the Stokes equation in a half-space. $J$. Evol. Equ., 1:115-142, 2001.

[8] X. T. Duong and D. W. Robinson. Semigroup kernels, Poisson bounds, and holomorphic functional calculus. J. Funct. Anal., 142(1):89-128, 1996.

[9] A. Friedman. Partial Differential Equations. Holt, Rinehart and Winston, 1969.

[10] E. Gagliardo. Ulteriori proprietà di alcune classi di funzioni in più variabili. Ricerche di Mat. Napoli, 8:24-51, 1959.

[11] Y. Giga. The nonstationary Navier-Stokes system with some first order boundary condition. Proc. Japan Acad. Ser. A Math. Sci., 58(3):101-104, 1982.

[12] Y. Giga. Solutions for semilinear parabolic equations in $L^{p}$ and regularity of weak solutions of the Navier-Stokes system. J. Differential Equations, 62(2):186-212, 1986.

[13] Y. Giga and H. Sohr. Abstract $L^{p}$ estimates for the Cauchy problem with applications to the Navier-Stokes equations in exterior domains. J. Funct. Anal., 102:72-94, 1991.

[14] G. Grubb and V.A. Solonnikov. Boundary value problems for the nonstationary NavierStokes equations treated by pseudo-differential methods. Math. Scand., 69(2):217-290, 1991. 
[15] P.W. Jones. Quasiconformal mappings and extendability of functions in Sobolev spaces. Acta Math., 147:71-88, 1981.

[16] N. Kalton and L. Weis. The $H^{\infty}$-calculus and sums of closed operators. Math. Ann., 321:319-345, 2001.

[17] T. Kato. Strong $L^{p}$-solutions of the Navier-Stokes equation in $\mathbf{R}^{m}$, with applications to weak solutions. Math. Z., 187(4):471-480, 1984.

[18] M. McCracken. The resolvent problem for the Stokes equation on halfspaces in $L_{p}$. SIAM J. Math. Anal., 12:201-228, 1981.

[19] A. McIntosh. Operators which have an $H^{\infty}$-calculus. Proc. Centre Math. Analysis, 14:210-231, 1986.

[20] T. Miyakawa. The $L^{p}$ approach to the Navier-Stokes equations with the Neumann boundary condition. Hiroshima Math. J., 10(3):517-537, 1980.

[21] L. Nirenberg. On elliptic partial differential equations. Ann. Scuola Norm. Sup. Pisa (Ser. III), 13:115-162, 1959.

[22] A. Noll and J. Saal. $H^{\infty}$-calculus for the Stokes operator on $L_{q}$-spaces. Math.Z., 244:651$688,2003$.

[23] J. Prüss and H. Sohr. Imaginary powers of elliptic second order differential operators in $L^{p}$-spaces. Hiroshima Math. J., 23:161-192, 1993.

[24] J. Saal. Robin Boundary Conditions and Bounded $H^{\infty}$-Calculus for the Stokes Operator. PhD thesis, TU Darmstadt. Logos Verlag, Berlin, 2003.

[25] J. Saal. The Stokes operator with Robin boundary conditions in $L_{\sigma}^{1}\left(\mathbf{R}_{+}^{n}\right)$ and $L_{\sigma}^{\infty}\left(\mathbf{R}_{+}^{n}\right)$. In preparation, 2003.

[26] V.A. Solonnikov. Estimates for solutions of nonstationary Navier-Stokes equations. $J$. Sov. Math., 8:467-529, 1977.

[27] E. M. Stein and G. Weiss. Introduction to Fourier Analysis on Euclidean Spaces. Princeton University Press, 1971.

[28] S. Ukai. A solution formula for the Stokes equation in $\mathbf{R}_{+}^{n}$. Comm. Pure Appl. Math., 40(5):611-621, 1987.

\section{Jürgen Saal}

Department of Mathematics

Hokkaido University

Sapporo, 060-0810, Japan

e-mail: saal@mathematik.tu-darmstadt.de 